\title{
Metabolic Profiling of the Oil of Sesame of the Egyptian Cultivar 'Giza 32' Employing LC-MS and Tandem MS-Based Untargeted Method
}

\author{
Reham Hassan Mekky 1,2,*(D), Essam Abdel-Sattar ${ }^{3}\left(\mathbb{D}\right.$, Antonio Segura-Carretero ${ }^{2,4}$ \\ and María del Mar Contreras $2,4,5, *$ (D)
}

check for updates

Citation: Mekky, R.H.; Abdel-Sattar, E.; Segura-Carretero, A.; Contreras, M.d.M. Metabolic Profiling of the Oil of Sesame of the Egyptian Cultivar 'Giza 32' Employing LC-MS and Tandem MS-Based Untargeted Method. Foods 2021, 10, 298. https: //doi.org/10.3390/foods10020298

Academic Editor: Charles Brennan Received: 28 December 2020

Accepted: 28 January 2021

Published: 2 February 2021

Publisher's Note: MDPI stays neutra with regard to jurisdictional claims in published maps and institutional affiliations.

Copyright: (C) 2021 by the authors Licensee MDPI, Basel, Switzerland. This article is an open access article distributed under the terms and conditions of the Creative Commons Attribution (CC BY) license (https:/ creativecommons.org/licenses/by/ $4.0 /)$
1 Department of Pharmacognosy, Faculty of Pharmacy, Egyptian Russian University, Badr City, Cairo-Suez Road, Cairo 11829, Egypt

2 Research and Development Functional Food Centre (CIDAF), Bioregión Building, Health Science Technological Park, Avenida del Conocimiento s/n, 18016 Granada, Spain; ansegura@ugr.es

3 Department of Pharmacognosy, Faculty of Pharmacy, Cairo University, El Kasr El-Aini Street, Cairo 11562, Egypt; essam.abdelsattar@pharma.cu.edu.eg

4 Department of Analytical Chemistry, Faculty of Sciences, University of Granada, Avenida Fuentenueva s/n, 18071 Granada, Spain

5 Department of Chemical, Environmental and Materials Engineering, Campus Las Lagunillas, Universidad de Jaén, 23071 Jaén, Spain

* Correspondence: reham-mekky@eru.edu.eg (R.H.M.); mcgamez@ujaen.es or mar.contreras.gamez@gmail.com (M.d.M.C.); Tel.: +20-100-5720-695 (R.H.M.)

Abstract: Sesame (Sesamum indicum L.) is a global oil crop. Sesame oil has been regarded as functional oil with antioxidant properties in several in vivo studies but little is known about its minor fraction. In this line, this study figures out the profile of the polar fraction of Egyptian cultivar Giza 32 sesame oil (SG32 oil) employing reversed-phase high-performance liquid chromatography coupled with diode array detection and electrospray ionization-quadrupole-time-of-flight-mass spectrometry and tandem MS. The characterization of the sesame oil metabolites depended on the observation of their retention time values, accurate MS, and MS/MS data, with UV spectra, and compared with relevant literature and available standards. Remarkably, 86 metabolites were characterized and sub-grouped into phenolic acids, lignans, flavonoids, nitrogenous compounds, and organic acids. From the characterized metabolites, 72 compounds were previously characterized in SG32 cake, which presented antioxidant properties, and hence it could contribute to SG32 oil antioxidant properties. Further studies are required to state the presence of such phenolics in commercial sesame oils and what of these compounds resist oil refining.

Keywords: sesame oil; Sesamum indicum L.; phenolic acids; lignans; flavonoids; core-shell columns; tandem MS

\section{Introduction}

Family Pedaliaceae belongs to the order Lamiales and consists of 14 genera and 70 species, including the famously known as sesame (Sesamum indicum L.) [1]. It is a cropproducing oil whose cultivation is distributed globally. On the base of total production, sesame seeds production is nearly 7 million tons with a production of 2 million tons of sesame oil [2]. Anciently, sesame originated from India. It was known in the Ancient Egyptian Civilization for the treatment of asthma since the third century BC [3-5]. Sesame seeds contain fats, proteins, carbohydrates, vitamins, and dietary fibers [5,6].

Besides their nutritional properties, several scientists explored possible biological activities and phytoconstituents of sesame seeds. In this sense, Dravie et al. [7] examined the antioxidant properties, total phenols, and flavonoids contents of Ghanaian sesame seeds via a multiple solvent extractions model. It was clear that the acetone extract was 
beyond most of the extracts in the antioxidant potential that were positively correlated with the total phenol content. As a matter of fact, sesame seeds have a myriad of biological activities, for instance, cardioprotective, hypolipidemic, hypocholesterolemic, anticancer, antioxidant, antidiabetic, and antihypertensive, among others [5,8-11]. Similarly, sesame seed oil has been recently regarded as functional oil with antioxidant properties [12,13]. In this line, Kim et al. [8] unraveled the auditory-protective effect of sesame oil in zebrafish and mice through the regulation of the hearing-related gene, Tecta. Concerning agri-food residues of sesame seeds, Khaleel et al. [14] have shown that residual sesame parts may exert some bioactivities.

Regarding the phytoconstituents retrieved from sesame, Dachtler et al. [15] characterized some furofurano lignans viz., sesamin, sesaminol, 6-episesaminol, and sesamolin using an online system of high-performance liquid chromatography (HPLC) hyphenated with nuclear magnetic resonance (NMR) and mass spectrometry (MS) detectors. In addition, Hassan [6] focused on the fatty acids composition of sesame oil. Moreover, $\mathrm{Wu}$ et al. [16] performed an LC-MS/MS combined with magnetic carboxylated multi-walled carbon nanotubes based-method for the determination of phenolic compounds in sesame oil. In another study, Görgüç et al. [17,18] incorporated vacuum and ultrasound-assisted enzymatic extraction to recover proteins and bioactive metabolites from sesame bran. Recently, Mekky et al. [10] performed untargeted metabolic profiling of sesame cake via reversed-phase (RP) (HPLC) — diode array detection (DAD) and hyphenated to electrospray ionization (ESI)-quadrupole-time-of-flight (QTOF)-MS and tandem MS. This technology enables the detection of phenolic acids, lignans, and flavonoids, giving an insight into the significance of such agri-food residue.

Similarly, this study aims at performing untargeted profiling of the metabolites of the polar fraction of sesame oil of the Egyptian cultivar 'Giza 32' (SG32) through RP-HPLCDAD-QTOF-MS and tandem MS to give new insights into the minor composition, with a focus on phenolic compounds. In fact, there is little information concerning the metabolic profiling of sesame oil, which has been limited to some phenolic classes and fatty acids, as commented before.

\section{Materials and Methods}

\subsection{Chemicals}

Solvents (n-hexane, methanol, acetonitrile, acetone, and glacial acetic acid) were obtained from Fisher Chemicals (Thermo Fisher Scientific, Waltham, MA, USA). They were of analytical and MS grade for extraction and characterization, respectively. A Milli-Q system (Millipore, Bedford, MA, USA) was used for obtaining ultrapure water. Standards were purchased from Sigma-Aldrich (St. Louis, MO, USA), except for some amino acids (L-tryptophan and L-phenylalanine), which were from Acros Organics (Morris Plains, NJ, USA). The degree of purity of all the used standards was around $95 \%(w / w)$.

\subsection{Samples Procurement and Oil Extraction Procedures}

SG32 seeds were identified and provided by Agriculture Engineer Nadia Abdel-Azim, Egyptian Ministry of Agriculture and Land Reclamation (Giza, Egypt). Firstly, they were ground into a fine powder with a particle size of around $1 \mathrm{~mm}$ via an Ultra Centrifugal Mill ZM 200, Retsch (Haan, Germany).

The extraction of SG32 seeds was according to Shyu and Hwang [19], with some modifications. The first step was to extract sesame oil through the homogenization of $1 \mathrm{~g}$ of SG32 seeds with $10 \mathrm{~mL} n$-hexane utilizing a magnetic stirrer Agimatic-N (Jp Selecta, Barcelona, Spain) for $30 \mathrm{~min}$ at room temperature followed by centrifugation at $7155 \times g$ at $5{ }^{\circ} \mathrm{C}$ for $15 \mathrm{~min}$ by a Sorvall ST 16 (Thermo Sci., ThermoFisher, Waltham, MA, USA). To recover oil fraction from SG32 seeds, $n$-hexane supernatant was collected and evaporated until dry under vacuum by a rotary evaporator at $38^{\circ} \mathrm{C}$ (Rotavapor R-200, Büchi Labortechnik, AG, Switzerland). As the second step and in order to extract the phenolic compounds from sesame oil, this fraction was reconstituted in $2 \mathrm{~mL} n$-hexane followed 
by $5 \mathrm{~mL}$ methanol:water $(80: 20, v / v)$ based on Ishtiaque et al. [20] to get the polar fraction of SG32 oil. The extraction mixture was agitated, centrifuged, and the supernatant collected. The previous step was repeated twice (methanol:water $(80: 20, v / v) \times 2.5 \mathrm{~mL}$ ) The methanolic-aqueous extracts were combined and defatted with $n$-hexane $(2 \mathrm{~mL})$ to eliminate any residual fat and concentrated using a speed-vacuum Concentrator plus (Eppendorf AG, Hamburg, Germany) at $30^{\circ} \mathrm{C}$ for above $3 \mathrm{~h}$. The polar oil fraction was appropriately dissolved in $2 \mathrm{~mL}$ of aqueous methanol $(80: 20, v / v)$ previously to subjection to RP-HPLC-DAD-ESI-QTOF-MS and -tandem MS analysis.

\subsection{Analysis by RP-HPLC-DAD-ESI-QTOF-MS and -Tandem MS}

The HPLC was an Agilent 1200 series equipped with a binary pump, an autosampler, and a diode array detector (DAD), (Agilent Technologies, Santa Clara, CA, USA) [21,22]. The separating column was a core-shell Halo C18 $(150 \mathrm{~mm} \times 4.6 \mathrm{~mm}, 2.7 \mu \mathrm{m}$ particle size, Advanced Materials Technologies, Wilmington, DE, USA). The system was hyphenated to a 6540 Agilent Ultra-High-Definition (UHD) Accurate-Mass Q-TOF LC/MS equipped with an Agilent Dual Jet Stream electrospray ionization (Dual AJS ESI) interface. MassHunter Workstation software (Agilent Technologies) was used for data acquisition $(2.5 \mathrm{~Hz})$ in profile mode. The spectra were acquired over a mass-to-charge $(\mathrm{m} / \mathrm{z})$ range from 70 to 1500 in negative-ion mode. The detection window was set to $100 \mathrm{ppm}$.

Data analysis was performed using MassHunter Qualitative Analysis B.06.00 (Agilent Technologies) according to $[10,21,23,24]$. In brief, compounds characterization was performed by observing candidates and the generation of their formulas within a mass error limit of $\pm 5 \mathrm{ppm}$. The MS score was set to $\geq 80$ [10]. The following databases were consulted: Reaxys [25],KNApSAcK Core System [26], SciFinder Scholar [27], PubChem [28], ChemSpider [29], METLIN Metabolite Database [30], Phenol-Explorer [31], the Dictionary of Natural Products [32], and Phytochemical dictionary of natural products database [33]. Moreover, relevant literature was traced via the Egyptian Knowledge Bank [34]. In addition, for characterization work validation, a comparison was made with standards whenever possible.

\section{Results and Discussion}

\subsection{RP-HPLC-DAD-ESI-QTOF-MS and Tandem-MS of SG32 Oil}

The phenolic fraction of SG32 oil was analyzed in negative ionization mode via core-shell RP-HPLC-DAD-ESI-QTOF-MS and tandem MS. Tables 1 and 2 classify the characterized metabolites into phenolics and non-phenolics. Besides, they demonstrate for each candidate the time (RT), experimental $\mathrm{m} / z$, generated molecular formulas, mass errors, scores, double bond equivalents (DBE), UV maxima (if present), tandem mass fragments, and relative abundance (area of chromatographic profiles of all characterized metabolites), respectively. This information was used for the characterization work, which was based on the strategy followed in our previous studies. Basically, the RT, molecular formula, and the fragmentation patterns were compared to those found in literature, databases, and standards, when possible. Moreover, fragmentation patterns enabled us to obtain clues about the functional groups, basic constituents, and/or polyphenol nucleus [10,23,35]. Nonetheless, further confirmation is required by NMR to also establish the stereochemistry.

Also, Tables S1 and S2 (supplementary material) mention metabolites classifications, and cite their previous description in the literature. A total of 86 metabolites were characterized with 11 metabolites reported for the first time in sesame, 59 metabolites observed for the first time in sesame oil, and 3 new proposed structures.

Moreover, Figure 1a represents the base peak chromatogram obtained by RP-HPLCDAD-ESI-QTOF-MS showing the complexity of the minor constituents of sesame oil as that for the seed cake (Figure 1c). 
Table 1. Phenolic compounds characterized in SG32 oil.

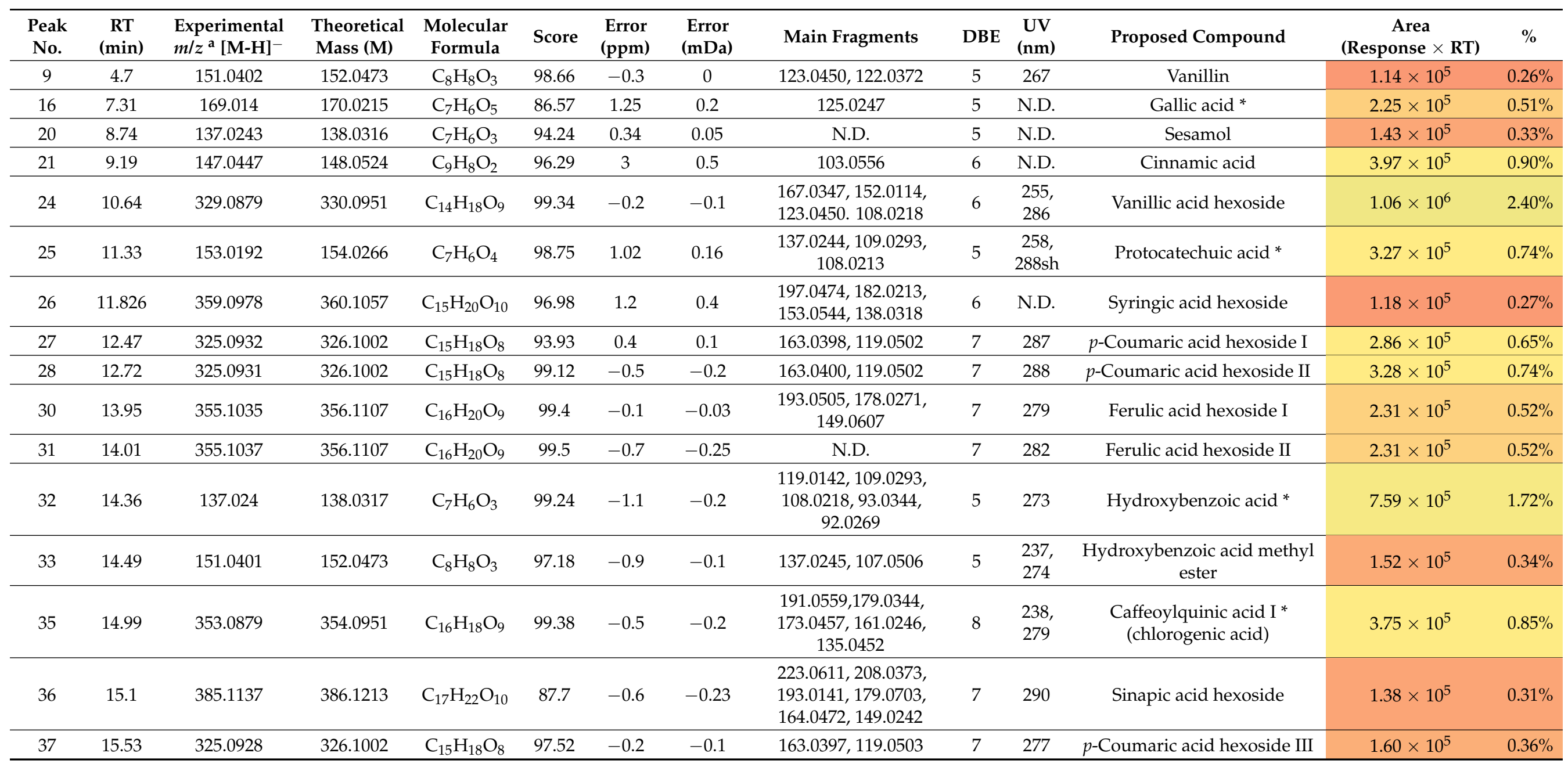


Table 1. Cont

\begin{tabular}{|c|c|c|c|c|c|c|c|c|c|c|c|c|c|}
\hline $\begin{array}{c}\text { Peak } \\
\text { No. }\end{array}$ & $\begin{array}{c}\text { RT } \\
(\mathrm{min})\end{array}$ & $\begin{array}{l}\text { Experimental } \\
m / z^{\text {a }}[\mathrm{M}-\mathrm{H}]^{-}\end{array}$ & $\begin{array}{l}\text { Theoretical } \\
\text { Mass (M) }\end{array}$ & $\begin{array}{l}\text { Molecular } \\
\text { Formula }\end{array}$ & Score & $\begin{array}{r}\text { Error } \\
(\mathrm{ppm})\end{array}$ & $\begin{array}{l}\text { Error } \\
(\mathrm{mDa})\end{array}$ & Main Fragments & DBE & $\begin{array}{l}\text { UV } \\
(\mathrm{nm})\end{array}$ & Proposed Compound & $\begin{array}{c}\text { Area } \\
(\text { Response } \times \text { RT })\end{array}$ & $\%$ \\
\hline 38 & 16.61 & 325.0929 & 326.1002 & $\mathrm{C}_{15} \mathrm{H}_{18} \mathrm{O}_{8}$ & 89.25 & -1.1 & -0.4 & N.D. & 7 & N.D. & $p$-Coumaric acid hexoside IV & $9.37 \times 10^{4}$ & $0.21 \%$ \\
\hline 39 & 16.68 & 355.1033 & 356.1107 & $\mathrm{C}_{16} \mathrm{H}_{20} \mathrm{O}_{9}$ & 99.7 & 0.5 & 0.18 & $\begin{array}{c}193.0500,178.0272 \\
149.0609\end{array}$ & 7 & 282 & Ferulic acid hexoside III & $9.21 \times 10^{5}$ & $2.09 \%$ \\
\hline 40 & 16.73 & 167.0346 & 168.0426 & $\mathrm{C}_{8} \mathrm{H}_{8} \mathrm{O}_{4}$ & 97.64 & 1.29 & 0.22 & $\begin{array}{c}152.0144,123.0449 \\
108.0251\end{array}$ & 5 & 258 & Vanillic acid * & $3.11 \times 10^{5}$ & $0.71 \%$ \\
\hline 41 & 17.11 & 593.151 & 594.1585 & $\mathrm{C}_{27} \mathrm{H}_{30} \mathrm{O}_{15}$ & 99.3 & 0.3 & 0.15 & $\begin{array}{c}503.1188,473.1085 \\
443.0981,413.0872, \\
383.0767,353.0667 \\
135.0457,119.0357 \\
117.0367\end{array}$ & 13 & $\begin{array}{l}267 \\
320\end{array}$ & $\begin{array}{l}\text { Luteolin C-deoxyhexoside } \\
\text { C-hexoside I }\end{array}$ & $1.42 \times 10^{6}$ & $3.22 \%$ \\
\hline 42 & 17.23 & 353.0876 & 354.0951 & $\mathrm{C}_{16} \mathrm{H}_{18} \mathrm{O}_{9}$ & 96.29 & 0.3 & 0.1 & $\begin{array}{c}191.0556,179.0327 \\
173.0450,161.0233 \\
135.0445\end{array}$ & 8 & 274 & Caffeoylquinic acid II & $2.52 \times 10^{5}$ & $0.57 \%$ \\
\hline 43 & 17.36 & 593.159 & 594.1585 & $\mathrm{C}_{27} \mathrm{H}_{30} \mathrm{O}_{15}$ & 99.3 & 0.3 & 0.15 & $\begin{array}{c}533.1285,503.1193 \\
473.1085,443.0989 \\
413.0873,383.0771 \\
353.0665\end{array}$ & 13 & $\begin{array}{l}270 \\
325\end{array}$ & $\begin{array}{c}\text { Luteolin C-deoxyhexoside } \\
\text { C-hexoside II }\end{array}$ & $1.59 \times 10^{6}$ & $3.60 \%$ \\
\hline 44 & 17.34 & 197.0453 & 198.05282 & $\mathrm{C}_{9} \mathrm{H}_{10} \mathrm{O}_{5}$ & 96.97 & 0.6 & 0.1 & N.D. & 5 & & Syringic acid * & $1.12 \times 10^{5}$ & $0.25 \%$ \\
\hline 45 & 17.47 & 355.1034 & 356.1107 & $\mathrm{C}_{16} \mathrm{H}_{20} \mathrm{O}_{9}$ & 96.4 & 0.4 & 0.16 & N.D. & 7 & 287 & Ferulic acid hexoside IV & $1.64 \times 10^{5}$ & $0.37 \%$ \\
\hline 46 & 17.78 & 289.0718 & 290.079 & $\mathrm{C}_{15} \mathrm{H}_{14} \mathrm{O}_{6}$ & 92.99 & 0.79 & 0.02 & $\begin{array}{c}253.0334,245.1390 \\
217.0027,131.0712 \\
123.0450\end{array}$ & 9 & N.D. & (-)-Epicatechin * & $2.52 \times 10^{5}$ & $0.57 \%$ \\
\hline 47 & 17.78 & 531.1719 & 532.1792 & $\mathrm{C}_{23} \mathrm{H}_{32} \mathrm{O}_{14}$ & 89.5 & -0.5 & -0.28 & $\begin{array}{c}179.0140,165.0554 \\
150.0317\end{array}$ & 8 & 283 & $\begin{array}{l}\text { Sinapic acid deoxyhexoside } \\
\text { hexoside }\end{array}$ & $1.46 \times 10^{5}$ & $0.33 \%$ \\
\hline 48 & 17.96 & 681.24 & 682.2473 & $\mathrm{C}_{32} \mathrm{H}_{42} \mathrm{O}_{16}$ & 99.63 & 0.54 & 0.37 & $357.1302,151.0384$ & 12 & $\begin{array}{l}242, \\
275\end{array}$ & Pinoresinol dihexoside I & $2.96 \times 10^{5}$ & $0.67 \%$ \\
\hline 49 & 18.21 & 563.1411 & 564.1479 & $\mathrm{C}_{26} \mathrm{H}_{28} \mathrm{O}_{14}$ & 99.03 & -0.57 & -0.32 & $\begin{array}{l}545.1297,503.1189, \\
473.1087,443.0979, \\
413.0872,383.0771, \\
353.0664,117.0343\end{array}$ & 13 & $\begin{array}{l}274 \\
330\end{array}$ & $\begin{array}{l}\text { Apigenin C-pentoside } \\
\text { C-hexoside I }\end{array}$ & $2.93 \times 10^{6}$ & $6.64 \%$ \\
\hline
\end{tabular}


Table 1. Cont.

\begin{tabular}{|c|c|c|c|c|c|c|c|c|c|c|c|c|c|}
\hline $\begin{array}{c}\text { Peak } \\
\text { No. }\end{array}$ & $\begin{array}{c}\text { RT } \\
(\mathrm{min})\end{array}$ & $\begin{array}{l}\text { Experimental } \\
m / z^{\text {a }}[\mathrm{M}-\mathrm{H}]^{-}\end{array}$ & $\begin{array}{l}\text { Theoretical } \\
\text { Mass (M) }\end{array}$ & $\begin{array}{l}\text { Molecular } \\
\text { Formula }\end{array}$ & Score & $\begin{array}{r}\text { Error } \\
(\mathrm{ppm})\end{array}$ & $\begin{array}{l}\text { Error } \\
(\mathrm{mDa})\end{array}$ & Main Fragments & DBE & $\begin{array}{c}\text { UV } \\
(\mathrm{nm})\end{array}$ & Proposed Compound & $\begin{array}{c}\text { Area } \\
(\text { Response } \times \text { RT) }\end{array}$ & $\%$ \\
\hline 50 & 18.27 & 121.0293 & 122.0368 & $\mathrm{C}_{7} \mathrm{H}_{6} \mathrm{O}_{2}$ & 99.78 & 1.2 & 0.15 & 92.0269 & 5 & 278 & Benzoic acid & $1.22 \times 10^{6}$ & $2.76 \%$ \\
\hline 51 & 18.63 & 563.1405 & 564.1479 & $\mathrm{C}_{26} \mathrm{H}_{28} \mathrm{O}_{14}$ & 98.83 & 0.08 & 0.05 & $\begin{array}{l}545.1323,503.1179, \\
473.1083,443.0976, \\
413.0871,383.0767, \\
353.0666,117.0335\end{array}$ & 13 & $\begin{array}{l}272 \\
327\end{array}$ & $\begin{array}{l}\text { Apigenin C-pentoside } \\
\text { C-hexoside II }\end{array}$ & $2.20 \times 10^{5}$ & $0.50 \%$ \\
\hline 52 & 18.89 & 563.1408 & 564.1479 & $\mathrm{C}_{26} \mathrm{H}_{28} \mathrm{O}_{14}$ & 98.83 & 0.08 & 0.05 & $\begin{array}{l}545.1298,503.1191, \\
473.1087,443.0976, \\
413.0878,383.0769, \\
353.0666,117.0336\end{array}$ & 13 & $\begin{array}{l}269 \\
331\end{array}$ & $\begin{array}{l}\text { Apigenin C-pentoside } \\
\text { C-hexoside III }\end{array}$ & $2.06 \times 10^{6}$ & $4.67 \%$ \\
\hline 53 & 18.87 & 447.0935 & 448.1006 & $\mathrm{C}_{21} \mathrm{H}_{20} \mathrm{O}_{11}$ & 99.41 & -0.46 & -0.21 & $\begin{array}{c}327.0515,179.0141 \\
135.0447 \\
\end{array}$ & 12 & $\begin{array}{l}268 \\
325\end{array}$ & Luteolin C-hexoside I & $2.94 \times 10^{5}$ & $0.67 \%$ \\
\hline 54 & 19.5 & 447.093 & 448.1006 & $\mathrm{C}_{21} \mathrm{H}_{20} \mathrm{O}_{11}$ & 91.66 & 0.28 & 0.21 & $\begin{array}{c}327.0539,179.0138 \\
135.0450\end{array}$ & 12 & N.D. & Luteolin C-hexoside II & $1.92 \times 10^{5}$ & $0.44 \%$ \\
\hline 55 & 20.34 & 151.04 & 152.0473 & $\mathrm{C}_{8} \mathrm{H}_{8} \mathrm{O}_{3}$ & 98.04 & -0.3 & 0 & $136.0167,92.0269$ & 5 & 256 & Methoxybenzoic acid I & $9.28 \times 10^{5}$ & $2.11 \%$ \\
\hline 56 & 20.4 & 163.0399 & 164.0473 & $\mathrm{C}_{9} \mathrm{H}_{8} \mathrm{O}_{3}$ & 97.35 & 0.47 & 0.08 & N.D. & 6 & N.D. & $p$-Coumaric acid * & $1.17 \times 10^{5}$ & $0.27 \%$ \\
\hline 58 & 20.59 & 593.151 & 594.1585 & $\mathrm{C}_{27} \mathrm{H}_{30} \mathrm{O}_{15}$ & 99.5 & 0.3 & 0.16 & $\begin{array}{c}447.0897,285.0396 \\
133.0281\end{array}$ & 13 & $\begin{array}{l}267 \\
320\end{array}$ & $\begin{array}{l}\text { Luteolin deoxyhexoside } \\
\text { hexoside }\end{array}$ & $1.65 \times 10^{5}$ & $0.37 \%$ \\
\hline 59 & 20.93 & 300.9988 & 302.00627 & $\mathrm{C}_{14} \mathrm{H}_{6} \mathrm{O}_{8}$ & 94.92 & 0.82 & 0.25 & N.D. & 12 & N.D. & Ellagic acid * & $2.68 \times 10^{4}$ & $0.06 \%$ \\
\hline 60 & 21.06 & 463.0874 & 464.09548 & $\mathrm{C}_{21} \mathrm{H}_{20} \mathrm{O}_{12}$ & 84.99 & 0.25 & 0.12 & $300.9980,151.0030$ & 12 & N.D. & $\begin{array}{c}\text { Quercetin } \\
\text { 3-O- } \beta \text {-D-galactopyranoside * }\end{array}$ & $5.82 \times 10^{4}$ & $0.13 \%$ \\
\hline 61 & 21.18 & 623.1972 & 624.2054 & $\mathrm{C}_{29} \mathrm{H}_{36} \mathrm{O}_{15}$ & 94.2 & 1.52 & 0.95 & $\begin{array}{c}461.1649,387.1502, \\
315.1074,297.0957, \\
\text { 179.0347, 161.0239, } \\
153.0543,135.0447 \\
113.0233\end{array}$ & 12 & N.D. & Verbascoside & $2.80 \times 10^{5}$ & $0.64 \%$ \\
\hline 62 & 21.18 & 681.2402 & 682.2473 & $\mathrm{C}_{32} \mathrm{H}_{42} \mathrm{O}_{16}$ & 99.38 & -0.12 & -0.08 & $\begin{array}{c}\text { 519.1525, 357.1333 } \\
179.0529,151.0382 \\
149.0467\end{array}$ & 12 & N.D. & Pinoresinol dihexoside II & $7.45 \times 10^{4}$ & $0.17 \%$ \\
\hline
\end{tabular}


Table 1. Cont

\begin{tabular}{|c|c|c|c|c|c|c|c|c|c|c|c|c|c|}
\hline $\begin{array}{l}\text { Peak } \\
\text { No. }\end{array}$ & $\begin{array}{c}\text { RT } \\
(\mathrm{min})\end{array}$ & $\begin{array}{l}\text { Experimental } \\
m / z^{\text {a }}[\mathrm{M}-\mathrm{H}]^{-}\end{array}$ & $\begin{array}{l}\text { Theoretical } \\
\text { Mass (M) }\end{array}$ & $\begin{array}{l}\text { Molecular } \\
\text { Formula }\end{array}$ & Score & $\begin{array}{l}\text { Error } \\
(\mathrm{ppm})\end{array}$ & $\begin{array}{l}\text { Error } \\
(\mathrm{mDa})\end{array}$ & Main Fragments & DBE & $\begin{array}{l}\text { UV } \\
(\mathrm{nm})\end{array}$ & Proposed Compound & $\begin{array}{c}\text { Area } \\
(\text { Response } \times \text { RT) }\end{array}$ & $\%$ \\
\hline 63 & 21.3 & 463.0883 & 464.09548 & $\mathrm{C}_{21} \mathrm{H}_{20} \mathrm{O}_{12}$ & 98.82 & -0.02 & -0.01 & $\begin{array}{c}301.0324,300.0248 \\
271.02228,255.0276, \\
178.9974,151.0027 \\
136.0172,135.0447\end{array}$ & 12 & $\begin{array}{l}250 \\
352\end{array}$ & $\begin{array}{c}\text { Quercetin } \\
\text { 3-O- } \beta \text {-D-glucopyranoside * }\end{array}$ & $9.70 \times 10^{5}$ & $2.20 \%$ \\
\hline 64 & 21.36 & 447.0936 & 448.1006 & $\mathrm{C}_{21} \mathrm{H}_{20} \mathrm{O}_{11}$ & 97.26 & -0.84 & -0.38 & $\begin{array}{c}285.0402,151.0026 \\
133.0284\end{array}$ & 12 & N.D. & $\begin{array}{c}\text { Luteolin } \\
\text { 7-O- } \beta \text {-D-glucopyranoside * }\end{array}$ & $1.27 \times 10^{6}$ & $2.89 \%$ \\
\hline 65 & 21.87 & 575.1189 & 576.1268 & $\mathrm{C}_{30} \mathrm{H}_{24} \mathrm{O}_{12}$ & 92.55 & 0.51 & 0.3 & N.D. & 19 & N.D. & Procyanidin A2* & $1.22 \times 10^{4}$ & $0.03 \%$ \\
\hline 66 & 21.79 & 1017.3111 & 1018.3165 & $\mathrm{C}_{44} \mathrm{H}_{58} \mathrm{O}_{27}$ & 96.98 & -1.71 & -1.74 & $\begin{array}{l}\text { 855.2573, 693.2019, } \\
369.0973,323.0977, \\
221.0642,219.0663, \\
179.0559,161.0452, \\
149.0451,143.0349\end{array}$ & 16 & 280 & Sesaminol tetrahexoside I & $1.86 \times 10^{5}$ & $0.42 \%$ \\
\hline 67 & 22.27 & 1017.3095 & 1018.3165 & $\mathrm{C}_{44} \mathrm{H}_{58} \mathrm{O}_{27}$ & 98.45 & -0.15 & -0.15 & $\begin{array}{c}855.2556,693.2026, \\
369.0971,323.0973, \\
221.0682,179.0555, \\
161.0459,149.0443, \\
143.0342\end{array}$ & 16 & 280 & Sesaminol tetrahexoside II & $3.13 \times 10^{5}$ & $0.71 \%$ \\
\hline 68 & 22.39 & 593.1504 & 594.1585 & $\begin{array}{c}\mathrm{C}_{27} \mathrm{H}_{30} \\
\mathrm{O}_{15}\end{array}$ & 96.05 & 0.84 & 0.5 & N.D. & 13 & N.D. & Kaempferol 3-O-rutinoside * & $7.10 \times 10^{4}$ & $0.16 \%$ \\
\hline 69 & 22.52 & 841.2775 & 842.2857 & $\mathrm{C}_{38} \mathrm{H}_{50} \mathrm{O}_{21}$ & 99.4 & -0.04 & -0.04 & $\begin{array}{c}679.2225,485.1504 \\
355.1176,323.0978 \\
221.0665,179.0548, \\
161.0454,149.0450 \\
143.0352,121.0288 \\
89.0245\end{array}$ & 14 & 286 & Xanthoxylol trihexoside & $7.38 \times 10^{5}$ & $1.68 \%$ \\
\hline 70 & 22.54 & 163.0404 & 164.0473 & $\mathrm{C}_{9} \mathrm{H}_{8} \mathrm{O}_{3}$ & 84.16 & -1.97 & -0.32 & N.D. & 6 & 284 & $m$-Coumaric acid * & $3.22 \times 10^{4}$ & $0.07 \%$ \\
\hline 71 & 22.6 & 193.0505 & 194.0579 & $\mathrm{C}_{10} \mathrm{H}_{10} \mathrm{O}_{4}$ & 99.6 & 0.5 & 0.1 & $\begin{array}{c}178.0270,134.0371 \\
119.0503\end{array}$ & 6 & $\begin{array}{l}230 \\
282 \\
310\end{array}$ & Ferulic acid * & $5.09 \times 10^{5}$ & $1.16 \%$ \\
\hline
\end{tabular}


Table 1. Cont

\begin{tabular}{|c|c|c|c|c|c|c|c|c|c|c|c|c|c|}
\hline $\begin{array}{c}\text { Peak } \\
\text { No. }\end{array}$ & $\begin{array}{c}\text { RT } \\
(\mathrm{min})\end{array}$ & $\begin{array}{l}\text { Experimental } \\
m / z^{\text {a }}[\mathrm{M}-\mathrm{H}]^{-}\end{array}$ & $\begin{array}{l}\text { Theoretical } \\
\text { Mass (M) }\end{array}$ & $\begin{array}{l}\text { Molecular } \\
\text { Formula }\end{array}$ & Score & $\begin{array}{r}\text { Error } \\
(\mathrm{ppm})\end{array}$ & $\begin{array}{r}\text { Error } \\
(\mathrm{mDa})\end{array}$ & Main Fragments & DBE & $\begin{array}{l}\text { UV } \\
(\mathrm{nm})\end{array}$ & Proposed Compound & $\begin{array}{c}\text { Area } \\
(\text { Response } \times \text { RT) } \\
\end{array}$ & $\%$ \\
\hline 72 & 22.73 & 855.2582 & 856.2637 & $\mathrm{C}_{38} \mathrm{H}_{48} \mathrm{O}_{22}$ & 96.7 & -1.2 & -1.55 & $\begin{array}{l}693.2036,485.1494, \\
369.0963,323.0999, \\
221.0663,179.0556, \\
161.0456,149.0446, \\
143.0346,119.0348\end{array}$ & 15 & 278 & Sesaminol trihexoside I & $1.14 \times 10^{6}$ & $2.59 \%$ \\
\hline 73 & 23.18 & 609.1822 & 610.1898 & $\mathrm{C}_{28} \mathrm{H}_{34} \mathrm{O}_{15}$ & 98.33 & 0.49 & 0.3 & $\begin{array}{c}447.1293,301.0713 \\
259.0811,175.0023 \\
151.0031\end{array}$ & 12 & 281 & $\begin{array}{l}\text { Hesperetin hexoside } \\
\text { deoxyhexoside }\end{array}$ & $2.63 \times 10^{5}$ & $0.60 \%$ \\
\hline 74 & 23.2 & 447.0943 & 448.1006 & $\mathrm{C}_{21} \mathrm{H}_{20} \mathrm{O}_{11}$ & 96.91 & -2.07 & -0.93 & $\begin{array}{c}285.0404,135.0452 \\
127.0764\end{array}$ & 12 & N.D. & $\begin{array}{c}\text { Kaempferol } \\
\text { 3-O- } \beta \text {-D-glucopyranoside * }\end{array}$ & $7.60 \times 10^{5}$ & $1.72 \%$ \\
\hline 75 & 23.3 & 855.2572 & 856.2637 & $\mathrm{C}_{38} \mathrm{H}_{48} \mathrm{O}_{22}$ & 98.3 & -0.6 & -0.53 & $\begin{array}{l}693.2060,485.1514, \\
369.0970,323.0983, \\
221.0667,179.0560, \\
161.0453,149.0452, \\
143.0352,119.0351\end{array}$ & 15 & 277 & Sesaminol trihexoside II & $5.46 \times 10^{5}$ & $1.24 \%$ \\
\hline 76 & 23.49 & 855.2568 & 856.2637 & $\mathrm{C}_{38} \mathrm{H}_{48} \mathrm{O}_{22}$ & 99.7 & -0.3 & -0.24 & $\begin{array}{l}693.2029,485.1508, \\
369.0981,323.0980, \\
221.0660,179.0563, \\
161.0457,149.0451, \\
143.0351,119.0349\end{array}$ & 15 & 287 & Sesaminol trihexoside III & $7.90 \times 10^{6}$ & $17.94 \%$ \\
\hline 78 & 24.21 & 871.2521 & 872.2586 & $\mathrm{C}_{38} \mathrm{H}_{48} \mathrm{O}_{23}$ & 94.5 & -0.7 & -0.61 & $\begin{array}{c}709.1983,691.1938, \\
485.1527,385.0925, \\
323.0985,221.0664, \\
179.0556,161.0447, \\
143.0354,137.0245, \\
119.0354,89.0245\end{array}$ & 15 & 277 & $\begin{array}{l}\text { Hydroxysesamolin } \\
\text { trihexoside }\end{array}$ & $4.42 \times 10^{5}$ & $1.00 \%$ \\
\hline
\end{tabular}


Table 1. Cont

\begin{tabular}{|c|c|c|c|c|c|c|c|c|c|c|c|c|c|}
\hline $\begin{array}{l}\text { Peak } \\
\text { No. }\end{array}$ & $\begin{array}{c}\mathrm{RT} \\
(\mathrm{min})\end{array}$ & $\begin{array}{l}\text { Experimental } \\
m / z^{\text {a }}[\mathrm{M}-\mathrm{H}]^{-}\end{array}$ & $\begin{array}{l}\text { Theoretical } \\
\text { Mass (M) }\end{array}$ & $\begin{array}{l}\text { Molecular } \\
\text { Formula }\end{array}$ & Score & $\begin{array}{l}\text { Error } \\
(\mathrm{ppm})\end{array}$ & $\begin{array}{l}\text { Error } \\
(\mathrm{mDa})\end{array}$ & Main Fragments & DBE & $\begin{array}{c}\text { UV } \\
(\mathrm{nm})\end{array}$ & Proposed Compound & $\begin{array}{c}\text { Area } \\
(\text { Response } \times \text { RT) }\end{array}$ & $\%$ \\
\hline 79 & 24.27 & 539.1775 & 540.1843 & $\mathrm{C}_{25} \mathrm{H}_{32} \mathrm{O}_{13}$ & 99.11 & -0.8 & -0.43 & $\begin{array}{c}377.1240,359.1192, \\
333.0842,327.0880, \\
275.0918,223.0641, \\
209.0455,191.0349, \\
179.0697,171.0293, \\
161.0473,153.0559, \\
127.0400\end{array}$ & 10 & $\begin{array}{l}235 \\
273\end{array}$ & $\begin{array}{c}\text { Sinapoyl-3-dehydroshikimic } \\
\text { acid hexoside I }\end{array}$ & $4.56 \times 10^{6}$ & $10.36 \%$ \\
\hline 81 & 24.43 & 679.2248 & 680.2319 & $\mathrm{C}_{32} \mathrm{H}_{40} \mathrm{O}_{16}$ & 98.53 & -0.35 & -0.24 & $\begin{array}{c}517.2748,485.1429, \\
355.1176,323.0964, \\
221.0661,179.0566, \\
161.0453,149.0449, \\
143.0342,121.0288, \\
89.0244 \\
\end{array}$ & 13 & 282 & Xanthoxylol dihexoside & $3.27 \times 10^{5}$ & $0.74 \%$ \\
\hline 82 & 24.76 & 539.1775 & 540.1843 & $\mathrm{C}_{25} \mathrm{H}_{32} \mathrm{O}_{13}$ & 99.09 & 0.52 & 0.28 & $\begin{array}{c}333.0854,327.0889, \\
223.0609 \text { 209.0446, } \\
191.0340,171.0299 \\
161.0482,153.0537 \\
127.0399\end{array}$ & 10 & $\begin{array}{l}230 \\
277\end{array}$ & $\begin{array}{c}\text { Sinapoyl-3- } \\
\text { dehydroshikimic acid } \\
\text { hexoside II }\end{array}$ & $2.46 \times 10^{5}$ & $0.56 \%$ \\
\hline 83 & 24.82 & 161.0249 & 162.0322 & $\mathrm{C}_{9} \mathrm{H}_{6} \mathrm{O}_{3}$ & 75.63 & -3.38 & -0.19 & N.D. & 7 & N.D. & $\begin{array}{l}\text { 7-hydroxycoumarin } \\
\text { (umbelliferone) * }\end{array}$ & $1.60 \times 10^{4}$ & $0.04 \%$ \\
\hline 84 & 24.97 & 151.04 & 152.0473 & $\mathrm{C}_{8} \mathrm{H}_{8} \mathrm{O}_{3}$ & 99.76 & -0.1 & 0 & $136.0167,92.0270$ & 5 & N.D. & Methoxybenzoic acid II & $2.41 \times 10^{5}$ & $0.55 \%$ \\
\hline 85 & 26.45 & 285.0408 & 286.0477 & $\mathrm{C}_{15} \mathrm{H}_{10} \mathrm{O}_{6}$ & 99.42 & -1.2 & -0.3 & $227.1288,135.0450$ & 11 & $\begin{array}{l}287 \\
325\end{array}$ & Luteolin * & $8.39 \times 10^{5}$ & $1.91 \%$ \\
\hline 86 & 28.32 & 271.0614 & 272.0685 & $\mathrm{C}_{15} \mathrm{H}_{12} \mathrm{O}_{5}$ & 99.03 & -0.7 & -0.2 & N.D. & 10 & N.D. & Naringenin * & $1.68 \times 10^{5}$ & $0.38 \%$ \\
\hline
\end{tabular}

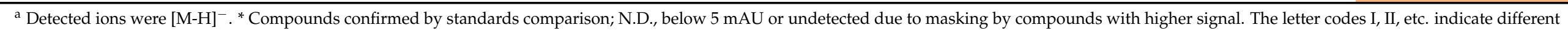
isomers. New proposed structures are in bold., DBE: double bond equivalents. Lowest value $\square$ Highest value. 
Table 2. Non-phenolic compounds characterized in SG32 oil.

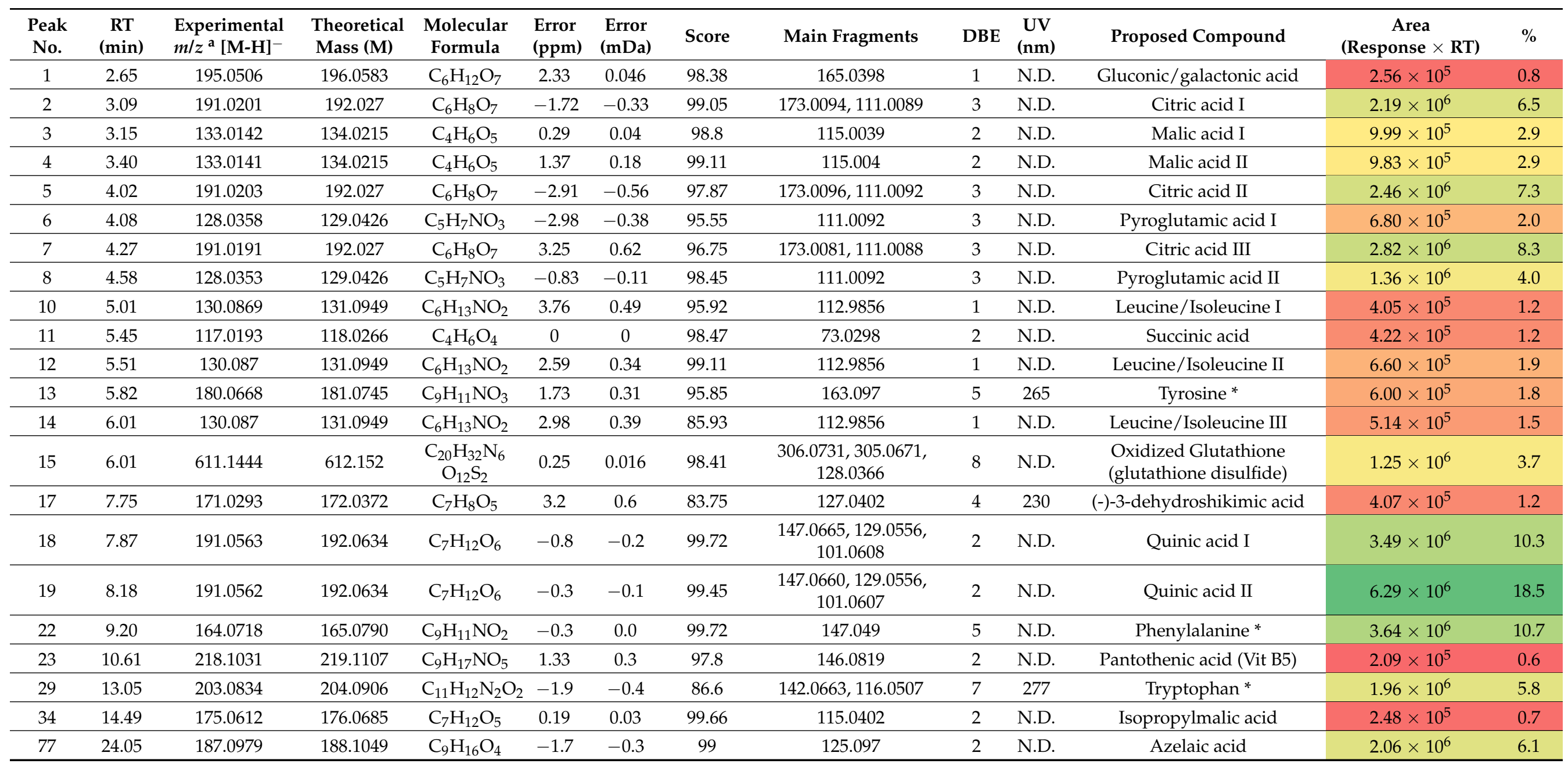

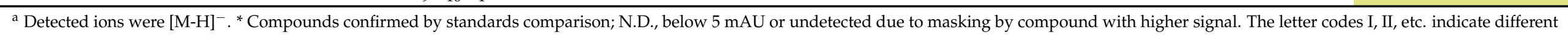
isomers. DBE: double bond equivalents. Lowest value $\square$ Highest value. 

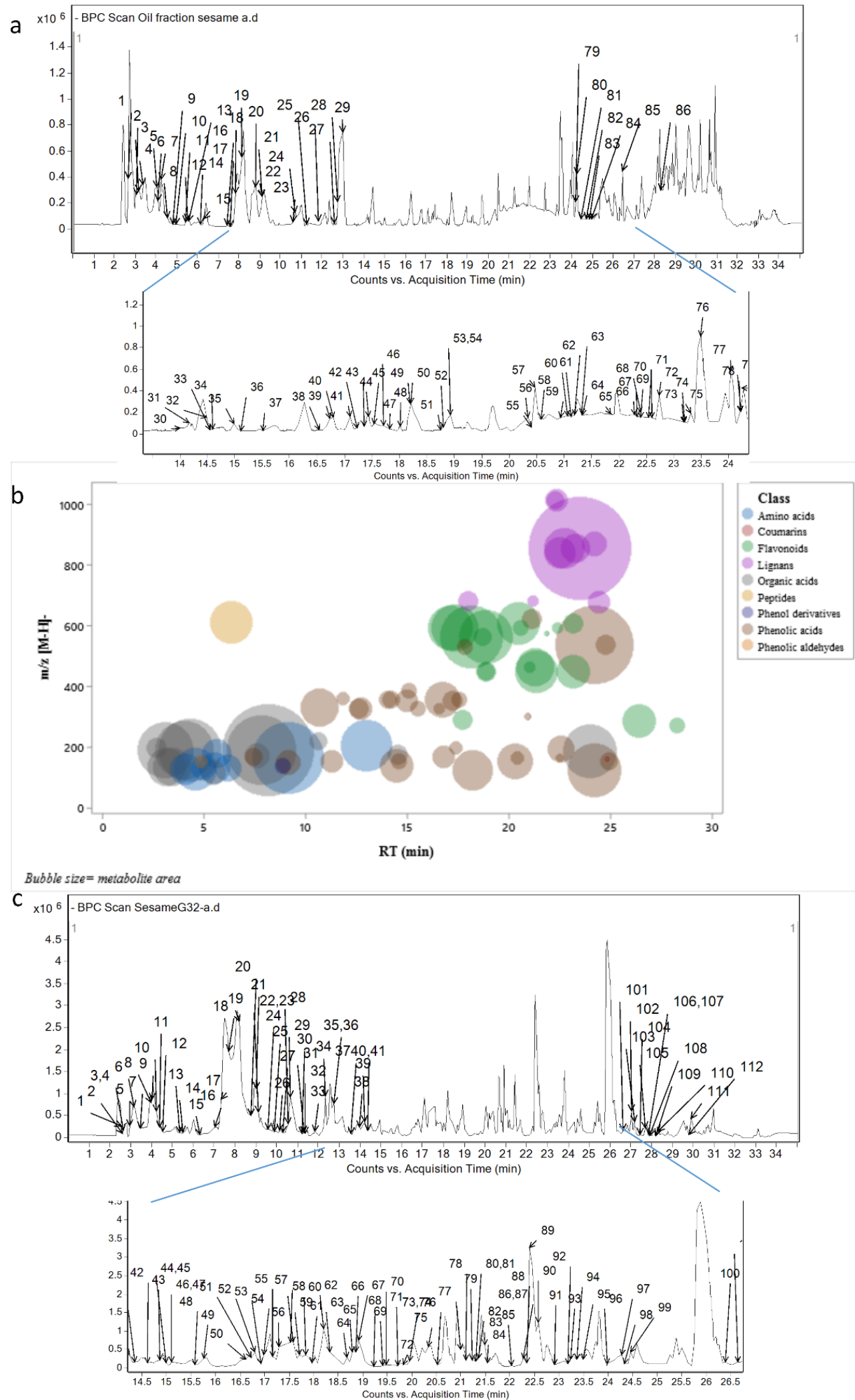

Figure 1. (a) Base peak chromatogram of SG32 oil and (b) its metabolites grouped into classes and classified according to $\mathrm{m} / \mathrm{z}$, retention time (RT), and relative area in SG32 oil. (c) Base peak chromatogram of SG32 cake, which has been adapted from [10]. 


\subsubsection{Phenolic Compounds}

Sixty-four phenolic compounds were observed in SG32 oil and could be categorized into phenolic acids (32), flavonoids (19), lignans (10), coumarins (1), phenol aldehydes (1), and phenol derivatives (1). Figure $1 \mathrm{~b}$ summarizes these compounds grouped into these phenolic classes, showing their position in the RP-HPLC-MS chromatogram and their abundance.

\section{Phenolic Acids}

The presence of phenolic acids was noticed with 32 phenolic compounds, being the major class of the annotated metabolites in qualitative and quantitative terms (Figure $1 \mathrm{~b}$, see Section 3.2). They belonged to three subclasses viz., hydroxybenzoic acids (12), hydroxycinnamic acids (19), and a hexahydroxydiphenic acid dilactone (Table 1 and Table S1).

Concerning hydroxybenzoic acids, compounds at $\mathrm{m} / \mathrm{z} 121.03$ and 135.05 showed the loss of $\mathrm{CO}(28 \mathrm{Da})$ and $\mathrm{CO}_{2}(44 \mathrm{Da})$, and $\lambda_{\max } 278$ and $273 \mathrm{~nm}$, respectively. They were described as benzoic acid and a methyl derivative [10]. Methylated and methoxy derivatives were also tentatively identified. In this sense, compounds at $m / z 151.40\left(\mathrm{C}_{8} \mathrm{H}_{8} \mathrm{O}_{3}\right)$ exerted neutral losses of methyl $\left(\mathrm{CH}_{3}, 15 \mathrm{Da}\right)$ followed by decarboxylation $\left(\mathrm{CO}_{2}, 44 \mathrm{Da}\right)$, which are typical of the presence of methoxy groups and phenolic acids, respectively. They were annotated as methoxybenzoic acid isomers I-II according to the Reaxys database. Figure 2a describes the main fragments of methoxybenzoic acid isomer II. Similarly, a methylated derivative was observed at RT $14.61 \mathrm{~min}$ and was characterized as hydroxybenzoic acid methyl ester. In this case, a loss of $\mathrm{CH}_{2}$ was observed instead of $\mathrm{CH}_{3}$ as in the aforementioned cases. Moreover, a mono-hydroxylated benzoic acid was also characterized, hydroxybenzoic acid $\left(m / z 137.02, \mathrm{C}_{7} \mathrm{H}_{6} \mathrm{O}_{3}\right)$, with the sequential loss of water and $\mathrm{CO}_{2}$ [21].

a

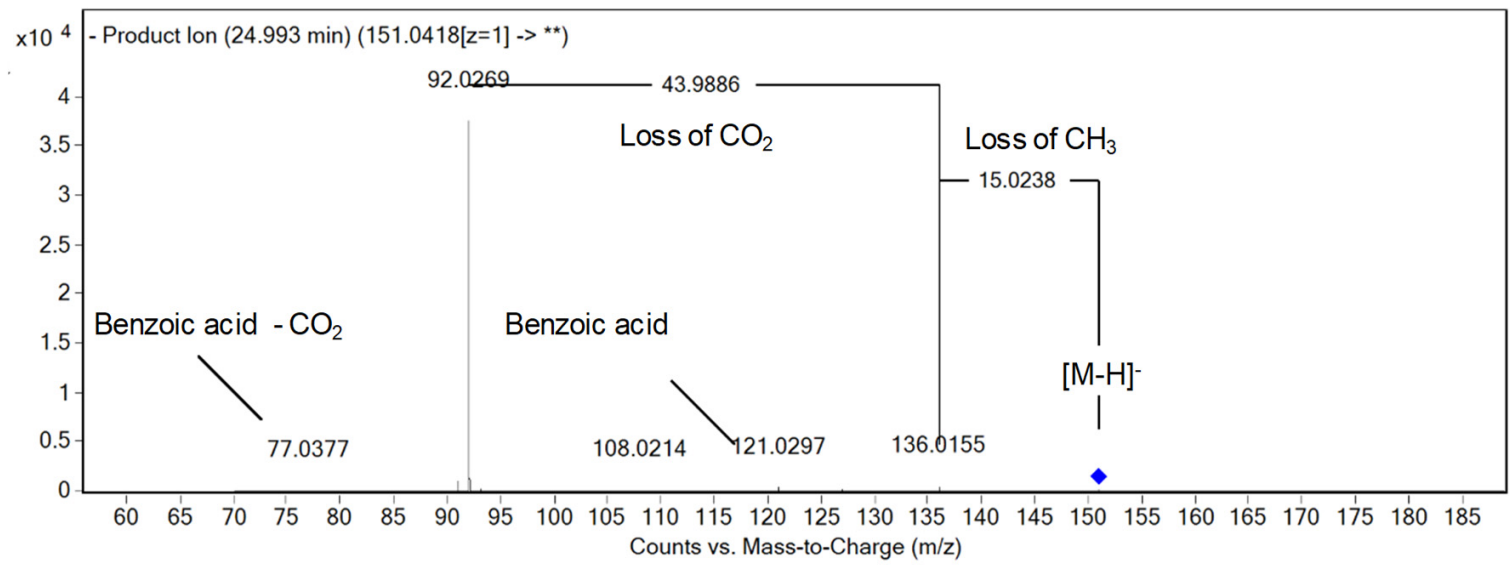

b

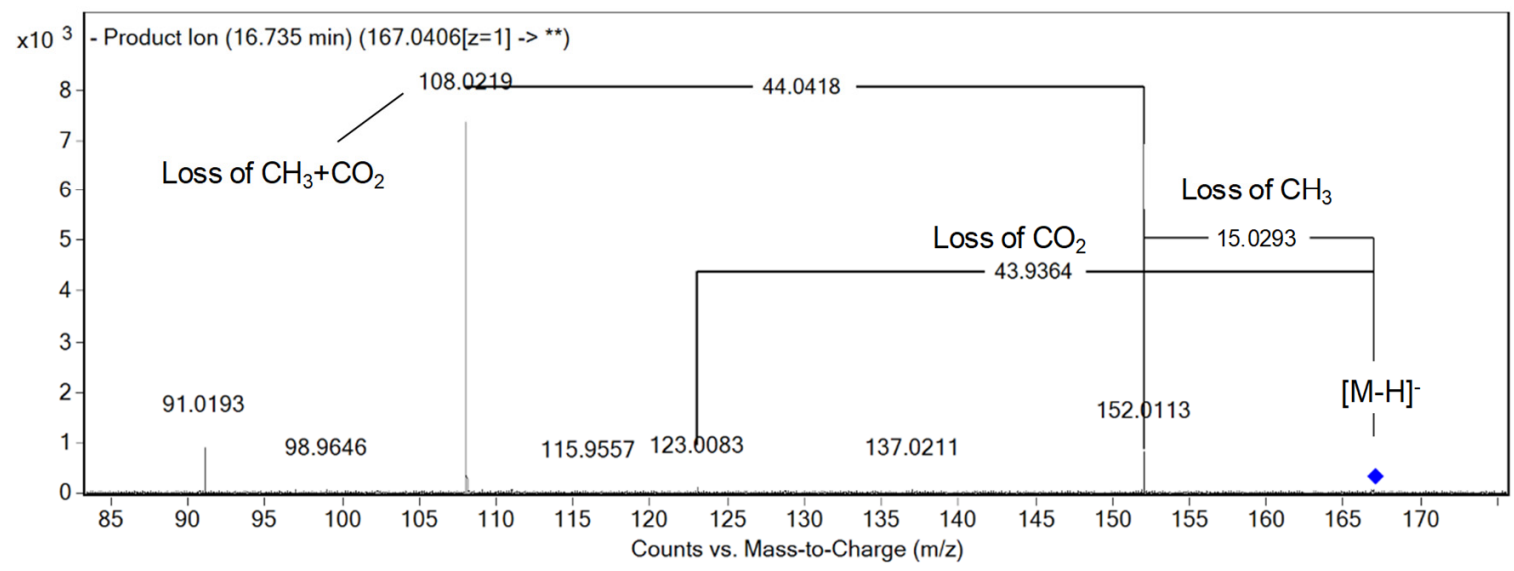

Figure 2. The patterns of fragmentation of (a) methoxybenzoic acid II and (b) vanillic acid. 
Dihydroxybenzoic acids were detected, and illustrated as, protocatechuic and vanillic acids (O-methylated derivative), at RT 11.26 and $16.79 \mathrm{~min}$, respectively. Both revealed the neutral loss of water $(18 \mathrm{Da})$ and $\mathrm{CO}_{2}$ with an additional loss of a methyl group $\left(\mathrm{CH}_{3}\right)$ in the case of vanillic acid [36] (Figure 2b). A glycosylated derivative of this compound (vanillic acid hexoside) was also characterized $\left(\mathrm{m} / \mathrm{z}\right.$ of $\left.329.09, \mathrm{C}_{14} \mathrm{H}_{18} \mathrm{O}_{9}\right)$, which showed a loss of a hexose (162 Da) as being linked through the hydroxyl moiety of the vanillic acid, as well as $\mathrm{CH}_{3}$ and $\mathrm{CO}_{2}$, as for the aforementioned phenolic acids. It bears noting that this is the first report of it in sesame oil [10].

Gallic and syringic acids presence (tri-hydroxylated benzoic acids) was confirmed with standards. Moreover, syringic acid hexoside was detected at (RT $11.81 \mathrm{~min}, \mathrm{~m} / \mathrm{z} 359.10$ ) showing the neutral loss of a hexose, demethylation, and decarboxylation, as before [10] (Table 1 and Table S1).

Concerning hydroxycinnamic acids, 19 derivatives were observed. They could be divided into cinnamic acid (non-hydroxylated), $p$-coumaric acid, and $m$-coumaric acid derivatives (mono-hydroxylated), caffeic acid, and ferulic acid derivatives (di-hydroxylated), and sinapic acid derivatives (tri-hydroxylated). It is noteworthy that the presence of $m$-coumaric, $p$-coumaric acid, chlorogenic (caffeoylquinic I), and ferulic acids was unambiguously confirmed with standards. Another isomer of caffeoylquinic acid was observed at RT $17.23 \mathrm{~min}$ with the presence of the fragment ions of quinic acid with its dehydrated ion $(m / z 191.0556$ and 173.0450) and caffeic acid with its dehydrated and decarboxylated ions $(m / z$ 179.0327, 161.0233, and 135.0455) [24]. Besides, the occurrence of $m$-coumaric acid and caffeoylquinic acids I-II is observed for the first time in sesame oil. In this line, a caffeoyl phenylethanoid derivative $\left(m / z 623.20, \mathrm{C}_{29} \mathrm{H}_{36} \mathrm{O}_{15}\right)$ was observed at RT $21.12 \mathrm{~min}$. The main detected fragments unraveled the neutral loss of a caffeoyl and a deoxyhexosyl moieties ( $m / z 461.17$ and 315.11) with the detection of caffeic acid ion and its dehydrated form (m/z 179.03 and 161.02). The hydroxytyrosol ion (phenylethanoid) was observed $(\mathrm{m} / \mathrm{z}$ 153.05) after the neutral loss of hexosyl moiety (Figure 3a) and so it is verbascoside according to previous studies $[10,24]$.

Additionally, peak $21\left(\mathrm{~m} / \mathrm{z}\right.$ of $\left.147.05, \mathrm{C}_{9} \mathrm{H}_{8} \mathrm{O}_{2}\right)$ showed the characteristic neutral loss of $44 \mathrm{Da}$ of phenolic acids and thus it was annotated as cinnamic acid [10] (Table 1, Table S1). Four isomers of $p$-coumaric acid hexosides were characterized at $m / z$ values of $325.09\left(\mathrm{C}_{15} \mathrm{H}_{18} \mathrm{O}_{8}\right)$ and with MS/MS revealing neutral losses of hexose, which release the aglycone nuclei $(m / z$ 163.04), followed by decarboxylation. Similar fragmentation patterns were obtained for four isomers of ferulic acid hexoside (peaks 30, 31, 39, and 45) [10]. Concerning tri-hydroxylated cinnamic acids, four sinapic acid derivatives were firstly detected in sesame oil. Briefly, sinapic acid hexoside was observed $(\mathrm{m} / \mathrm{z} 385.11$, $\mathrm{C}_{17} \mathrm{H}_{22} \mathrm{O}_{10}$ ) with neutral loss of the hexosyl moiety (162 Da) followed by the common fragments of sinapic acid [10]. In the same manner, sinapic acid deoxyhexoside hexoside was annotated at RT 17.83 min with the aforementioned fragmentation pattern and the additional loss of deoxyhexose. Finally, two undescribed sinapoyl-dehydroshikimic acid hexosides were detected, showing the neutral loss of a hexose $(\mathrm{m} / \mathrm{z} 377.12)$ with subsequent sinapic acid ion $(m / z 223.06)$ and the sequential loss of a methyl group $(m / z 209.0455)$ and water $(m / z$ 191.03). Moreover, dehydroshikmic acid ion was detected with $m / z 171.03$ followed by its dehydrated $(\mathrm{m} / \mathrm{z}$ 153.0537) and decarboxylated $(\mathrm{m} / \mathrm{z}$ 127.04) forms (Table 1 and Table S1), Figure $3 \mathrm{~b}$ shows the detailed fragmentation pattern.

In line with hexahydroxydiphenic acid dilactone, ellagic acid was observed upon comparison with a standard at RT $20.93 \mathrm{~min}$.

\section{Flavonoids}

The presence of flavonoids in SG32 oil was widely observed with 19 derivatives. They are sub-grouped into flavonols (5), flavones (10), a flavan-3-ol, a proanthocyanidin, and flavanones (2) (Table 1). It is noteworthy that the detection of kaempferol 3-O$\beta$-D-glucopyranoside, kaempferol 3-O-rutinoside, quercetin 3-O- $\beta$-D-glucopyranoside, quercetin 3-O-rhamnopyranoside, quercetin 3-O-rutinoside, luteolin, luteolin 7-O- $\beta$-D- 
glucopyranoside, (-)-epicatechin, procyanidin A2, and naringenin was based on standards comparison. They were all mentioned for the first time in sesame oil, while procyanidin A2 was also described for the first time in Pedaliaceae. This compound is found in other families like Ericaceae [37]. Figure 4a,b shows examples of the fragmentation patterns, highlighting the typical losses of hexose of $O$-glycosylated compounds.

a
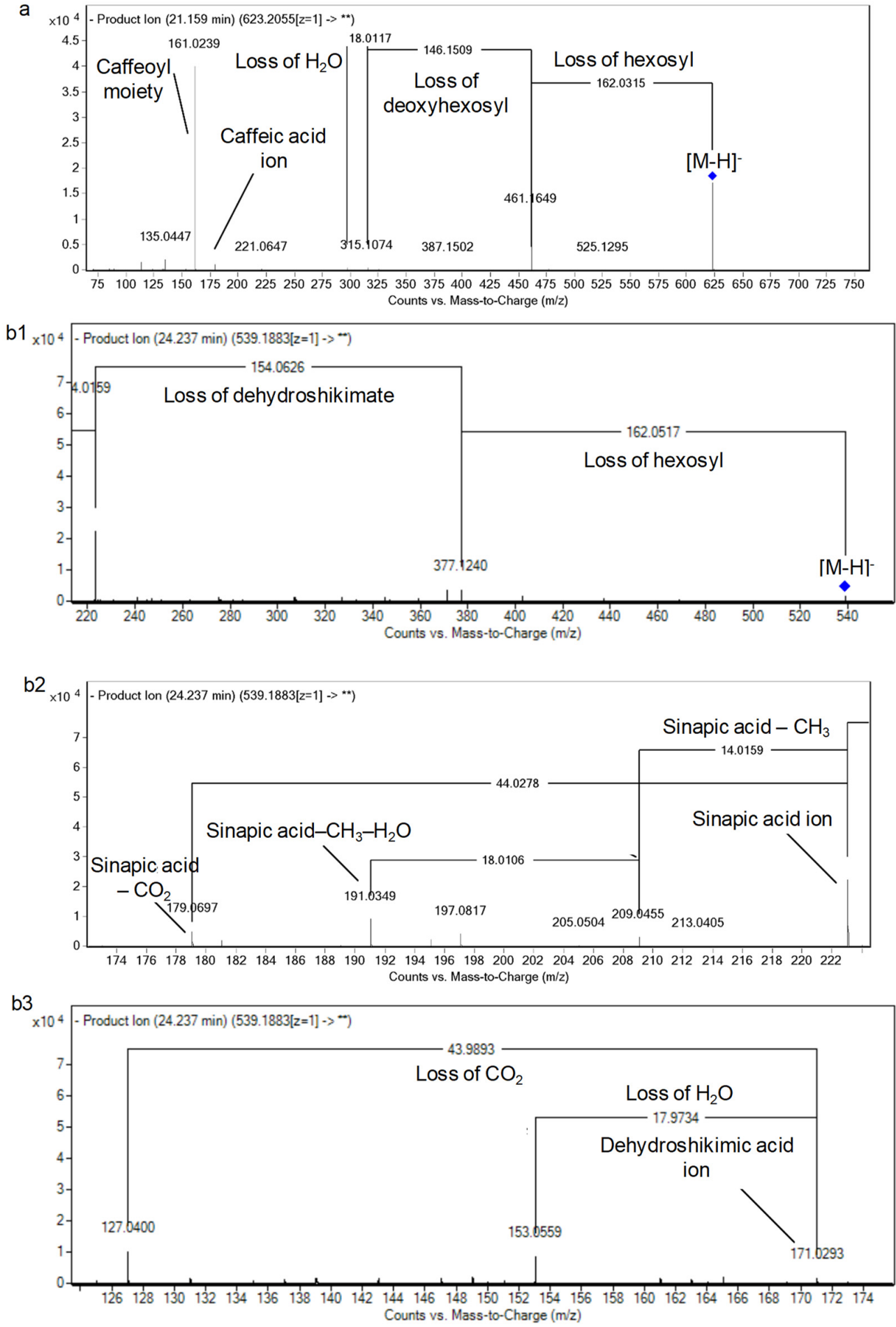

Figure 3. The patterns of fragmentation of (a) verbascoside, (b) sinapoyl-3-dehydroshikimic acid hexoside I. This spectrum has been divided (b1-b3) for a better description of the fragments. 

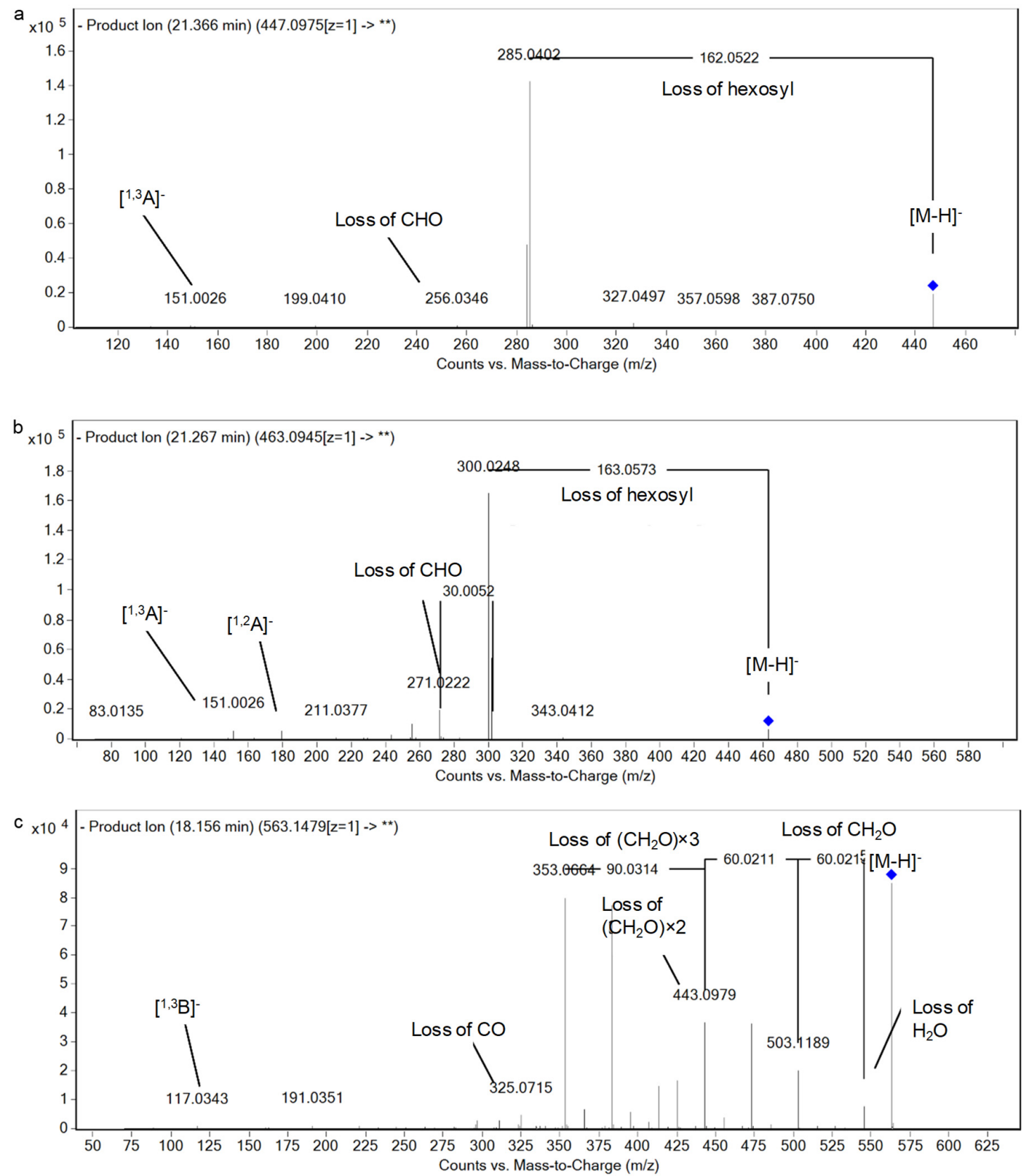

Figure 4. The patterns of fragmentation of (a) luteolin 7-O- $\beta$-D-glucopyranoside, (b) quercetin 3-O- $\beta$-D-glucopyranoside, and (c) apigenin C-pentoside C-hexoside I.

A similar fragmentation pattern to quercetin derivatives was observed for hesperetin hexoside deoxyhexoside (RT $23.18 \mathrm{~min}, \mathrm{~m} / z 609.18, \mathrm{C}_{28} \mathrm{H}_{34} \mathrm{O}_{15}$ ), with sequential losses of a hexosyl (162 Da, $m / z$ 447.13) and a deoxyhexosyl (146 Da, $m / z$ 301.07) from the O-hexoside and deoxyhexoside moieties. The fragmentation pattern of the aglycone unraveled the common fragment ion of $m / z 259.08$ for ( $\left.\mathrm{M}-\mathrm{H}-\mathrm{CH}_{2} \mathrm{CO}\right)$ followed by the ions at $m / z 175.00\left({ }^{0,4} \mathrm{~B}^{-}\right)$ and $151.00\left({ }^{1,3} \mathrm{~A}^{-}\right)$[38]. Furthermore, UV absorbance $\lambda_{\max } 281 \mathrm{~nm}$ indicated a flavanone structure. As far as we know, it is the first description of it in Pedaliaceae [39].

Mostly, flavones were represented in SG32 oil as C-glycosides conjugates of either apigenin or luteolin with the typical fragmentation of $\mathrm{C}$-glycosides. This is characterized by the loss of $18 \mathrm{Da}\left(\mathrm{H}_{2} \mathrm{O}\right), 44 \mathrm{Da}\left(\mathrm{CO}_{2}\right), 60 \mathrm{Da}\left(2 \times\left(\mathrm{CH}_{2} \mathrm{O}\right)\right), 90 \mathrm{Da}\left(3 \times\left(\mathrm{CH}_{2} \mathrm{O}\right)\right)$, and/or $120 \mathrm{Da}\left(4 \times\left(\mathrm{CH}_{2} \mathrm{O}\right)\right)$, according to previously reported studies $[10,40]$. In this line, apigenin $C$-pentoside $C$-hexoside (I-III) isomers were noticed exerting fragments at $\mathrm{m} / \mathrm{z}$ values of $541.13,503.12,473.11,443.10,383.08$, and 353.07 , and with the common ion 
at $m / z 117.03\left(1,3 \mathrm{~B}^{-}\right)$, Figure $4 \mathrm{c}[21,38,39,41]$. As for luteolin derivatives, two isomers of luteolin $C$-hexoside I-II and luteolin $C$-deoxyhexoside-C-hexoside I-II were annotated being characterized by a similar fragmentation pattern to the aforementioned apigenin derivatives and comparison with reported studies [10,42]. To our knowledge, this is the first report of apigenin and luteolin derivatives in sesame oil. Another luteolin derivative, but $O$-glycosylated, was observed at $m / z 593.15\left(\mathrm{C}_{27} \mathrm{H}_{30} \mathrm{O}_{15}\right)$ and thus it showed the neutral loss of a deoxyhexosyl $(\mathrm{m} / \mathrm{z} 447.09)$ and a hexosyl $(\mathrm{m} / \mathrm{z} 285.04)$ moieties. The aglycone also revealed fragment 133.03 suggesting the ion $\left(1,3 \mathrm{~B}^{-}\right)$and hence was described as luteolin deoxyhexoside hexoside, which was not reported before in genus Sesamum, as far as we know.

\section{Lignans}

Lignans are dimeric $\beta$ - $\beta^{\prime}$-linked phenylpropanoid compounds that are widely distributed in Kingdom Plantae and possess several biological activities [43]. Ten lignan derivatives were observed in SG32 oil. All of them are classified as furofuran lignans and they occurred as sugars conjugates where the loss of sugars was observed and the aglycones analogs were compared with previously reported studies [10,43,44]. Concisely, two isomers of pinoresinol dihexoside were annotated exerting the neutral loss of two hexosyl moieties $(\mathrm{m} / \mathrm{z} 357.1302,2 \times 162 \mathrm{Da})$ with an aglycone fragmentation showing an $\mathrm{m} / \mathrm{z}$ of 151 resulted from the cleavage of the tetrahydrofuran ring complying with earlier reports [10,43-45] (Table 1 and Table S1). Similarly, the ion $\mathrm{m} / z$ of 841.28 (RT $22.52 \mathrm{~min}, \mathrm{C}_{38} \mathrm{H}_{50} \mathrm{O}_{21}$.) exhibited a neutral loss of three hexosyl moieties consecutively, leading the fragment ions $\mathrm{m} / \mathrm{z}$ 679.22, 458.15, and 355.12). Besides, the fragments $\mathrm{m} / \mathrm{z} 161$ and $\mathrm{m} / \mathrm{z} 149$ were noticed standing for 1,3-dioxymethylenephenyl- $\mathrm{CHCHCH}_{2}$ and 1,3-dioxymethylenephenyl-CO, respectively [44]. Consequently, it was tentatively characterized as xanthoxylol trihexoside. Correspondingly, the unreported analog xanthoxylol dihexoside was observed at RT 24.46 min with a similar fragmentation pattern (Table 1 and Table S1, Figure 5a).

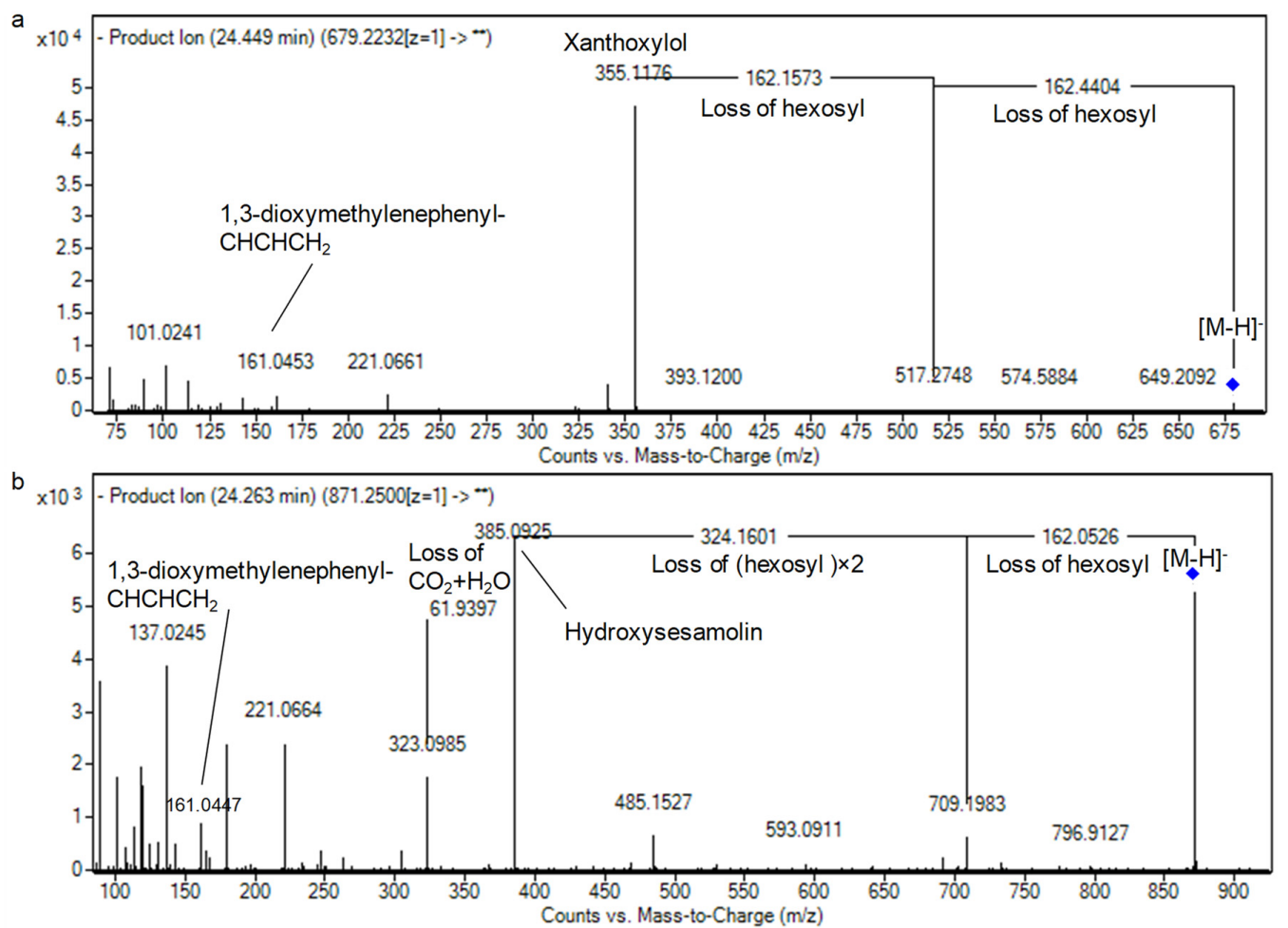

Figure 5. The patterns of fragmentation of (a) xanthoxylol dihexoside, and (b) hydroxysesamolin trihexoside.

About sesaminol, the presence of sesaminol trihexoside (I-III) and sesaminol tetrahexoside (I-II) isomers was noticed. As before, they were characterized by the corresponding 
losses of hexosyl moieties with the appearance of sesaminol aglycone $(\mathrm{m} / \mathrm{z}$ 369.10). Besides, the latter aglycone exhibited the fragments of $\mathrm{m} / z 161$ and $\mathrm{m} / \mathrm{z} 149$ complying with furofurano lignans $[10,46]$. Moreover, hydroxysesaminol trihexoside was detected as being characterized by the ion of the aglycone $\mathrm{m} / \mathrm{z} 385$ with the latter characteristic ion at $\mathrm{m} / \mathrm{z} 161$ (Figure $5 \mathrm{~b}$ ). All these lignans glycosides have been observed for the first time in sesame oil.

Coumarins, Phenol Aldehydes, and Derivatives

Besides the aforementioned classes, the coumarin 7-hydroxycoumarin (umbelliferone) presence was unambiguously confirmed upon comparison with a standard, while sesamol ( $m / z$ 137.02) [10] and vanillin (phenol aldehyde) were tentatively characterized [47].

\subsubsection{Non-Phenolic Compounds}

Nitrogenous Compounds

Concerning nitrogenous compounds, the occurrence of amino acids was observed by eight derivatives namely pyroglutamic acid (I-II) leucine/isoleucine (I-III), tyrosine, phenylalanine, and tryptophan. They exhibited the neutral loss of ammonia (17 Da) and/or $\mathrm{CO}_{2}(44 \mathrm{Da})$ complying with several reports $[10,21,24,36,45]$. Besides, the aromatic amino acids phenylalanine, tyrosine, and tryptophan were confirmed with standards. It bears noting that this is the first report of pyroglutamic acid in Pedaliaceae (Table 2 and Table S2). In this line, oxidized glutathione (GSSG) was detected (RT $6.32 \mathrm{~min}, \mathrm{~m} / \mathrm{z}$ 611.14) showing both glutathione $(\mathrm{m} / \mathrm{z}$ 306.08) and glutamyl $(\mathrm{m} / \mathrm{z}$ 128.04) moieties [10] and hence giving a clue of the occurrence of reduced glutathione (GSH) in SG32 oil being susceptible to conversion to GSSG during sampling and analysis [48]. As a matter of fact, GSH is a natural cellular antioxidant that prevents the onset and progression of many serious diseases [48]. This is the first report of GSSG in sesame oil and its presence in sesame oil provides a new aspect of its functionality (Table 2 and Table S2).

\section{Organic Acids}

Thirteen organic acids were detected in SG32 oil viz., gluconic/galactonic, citric (I-III), malic (I-II), succinic, (-)-3-dehydroshikimic, quinic (I-II), pantothenic, isopropylmalic, and azelaic acids. Their characterization are according to earlier reports $[10,24,36,47,49,50]$. All of them, as far as we know, were not reported before in sesame oil (Table 2 and Table S2).

\subsection{Semi-Quantitative Analysis}

Semi-quantitative analysis was performed via estimation of the total peak area obtained by MS denoting the relative amount of each characterized metabolite. In the perspective of subclasses of phenolic metabolites, phenolic acids subclass was the most abundant with (38.4\%) followed by flavonoids (33.7\%) then lignans (26.9\%) (Figure 6).

a

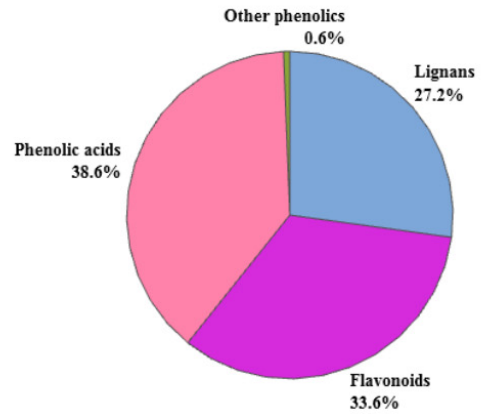

b

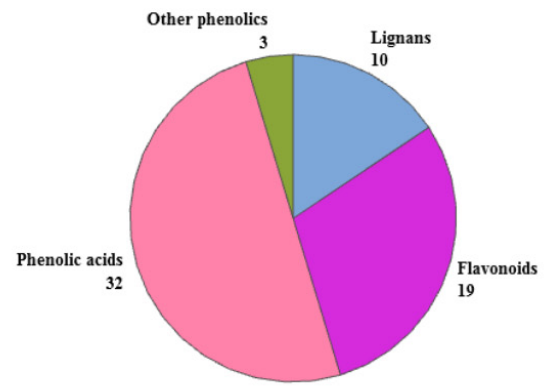

Figure 6. Phenolic compounds characterized in SG32 oil: (a) Relative abundance (\%) and (b) qualitative classification (\%). 
Concerning individual phenolic compounds, sesaminol trihexoside III (18\%), followed by sinapoyl-3-dehydroshikimic acid hexoside I ( $10 \%)$, apigenin $C$-pentoside $C$-hexoside I (7\%), methyl benzoic acid (5\%), and apigenin C-pentoside C-hexoside III $(5 \%)$ were the most abundant phenolic metabolites. In this context, $C$-glycosides are considered antioxidant capacity enhancers as the hydroxyl group and metal chelation sites in flavones are free $[24,41]$ and so it is expected that these phenolic compounds can protect the oil from oxidation.

\subsection{Comparison between Sesame Seed Oil and Cake}

Our previous study on SG32 cake counterpart exhibited the presence of 112 metabolites [10]. They belonged to the same classes of phytoconstituents in SG32 oil. Remarkably, 72 metabolites were detected in both SG32 cake and oil (Tables S1 and S2). The remaining metabolites in SG32 oil consist of generally minor phenolic compounds, and no sugars were observed as in the cake [10]. All the characterized lignans were of furofurano type, as in the seed cake. GSSG was detected in both samples.

Basically, sesame oil is considered a functional oil $[12,13]$. Since SG32 cake showed antioxidant activities, the common phenolic compounds between SG32 cake and oil could contribute to the biological potential of the oil. The phenolic profile in vegetable oils depends on the source and the processing. For example, olive oil is rich in hydroxytyrosol derivatives, among other phenolic compounds. Some of these compounds come from the olive fruit and pass to the oil, which is obtained by milling, malaxation, and centrifugation [51]. Nonetheless, the processing conditions affect the profile and the content of phenolic compounds in olive oil and thus its oxidative stability and shelf life [52,53]. Another example is tea seed oil obtained by screw pressing extraction, which also present tea phenolic compounds [54] and they contribute to the antioxidant stability [55]. In this context, it is important to establish the phenolic profile in vegetable oils due to the contribution of phenolic compounds to the functional and antioxidative properties of the vegetable oils. Therefore, other studies should be performed to address the phenolic composition integrity of virgin cold-pressed sesame oil and refined oil as industrial processes could lead to the loss or modification of phenolics compounds $[56,57]$. The employment of the state of art hyphenated techniques as U/HPLC, like in the current work, and/or GC coupled to high-resolution MS could help in this purpose as other authors have shown for other vegetable oils $[51,53,54,58]$. Even more, the data obtained here can be the basis for those characterization studies on sesame oils when using U/HPLC-MS. In fact, lignan aglycones have not been found in this work. It seems that lignans can be transformed during the oil production process, and some of them, like sesamolin and sesamin, are unstable [59].

\section{Conclusions}

In the present study, core-shell RP-HPLC-DAD-ESI-QTOF-MS and -MS/MS were utilized for the analysis of the oil of the Egyptian cultivar of sesame 'Giza 32'. Collectively, 86 metabolites were characterized in sesame SG32 oil, with 64 phenolic compounds with 3 unreported metabolites. The observed phenolic compounds were classified into phenolic acids, flavonoids, lignans, and others. All the characterized lignans were of furofurano type, as in the seed cake. Moreover, this is the first report showing oxidized glutathione in sesame oil. Mostly, the phenolic metabolites and other phytoconstituents in SG32 oil were present in SG32 cake counterpart, suggesting that they can pass from the seed cake to the oil, while in some cases the oil counterpart seems to be enriched. Consequently, further studies are required to detect the presence of important bioactive metabolites in commercial samples.

Supplementary Materials: The following are available online at https:/ /www.mdpi.com/2304-815 8/10/2/298/s1, Table S1: Phenolic compounds characterized in SG32 oil, Table S2: Non-phenolic compounds characterized in Sg32 oil. Gradient Elution and operating conditions by RP-HPLC-DADESI-QTOF-MS/MS. 
Author Contributions: Investigation, R.H.M. and M.d.M.C.; methodology, M.d.M.C.; data analysis, R.H.M.; validation, R.H.M. and M.d.M.C.; writing-original draft, R.H.M.; writing-review and editing; M.d.M.C., E.A.-S. and A.S.-C.; supervision, M.d.M.C., E.A.-S. and A.S.-C. All authors have read and agreed to the published version of the manuscript.

Funding: This work was supported by the International Cooperation Cell ICC06 under the Erasmus Mundus-Al Idrisi II programme "scholarship scheme for exchange and cooperation between Europe and North Africa". M.d.M.C. thanks the FEDER UJA project 1260905 funded by "Programa Operativo FEDER 2014-2020" and "Consejería de Economía y Conocimiento de la Junta de Andalucía".

Data Availability Statement: Part of the data presented in this study is available in Supplementary Material. Other data are available on request from the corresponding author.

Acknowledgments: The authors thank Agriculture Engineer Nadia Abdel-Azim, Egyptian Ministry of Agriculture and Land Reclamation (Giza, Egypt) for identifying and providing the plant material.

Conflicts of Interest: The authors declare no conflict of interest.

\section{References}

1. Berry, P.E. Lamiales. In Encyclopædia Britannica; Encyclopædia Britannica Inc.: Chicago, IL, USA, 2017.

2. $\quad$ FAO-Statistics. Productions, Crops; FAO: Rome, Italy, 2018.

3. Aboelsoud, N.H. Herbal medicine in ancient Egypt. J. Med. Plants Res. 2010, 4, 082-086.

4. Bedigian, D.; Harlan, J.R. Evidence for cultivation of sesame in the ancient world. Econ. Bot. 1986, 40, 137-154. [CrossRef]

5. Lim, T. Sesamum indicum. In Edible Medicinal and Non-Medicinal Plants; Springer: Dordrecht, The Netherlands, 2012; Volume 4, pp. 187-219.

6. Hassan, M.A. Studies on Egyptian Sesame Seeds (Sesamum indicum L.) and Its Products 1-Physicochemical Analysis and Phenolic Acids of Roasted Egyptian Sesame seeds (Sesamum indicum L.). World J. Dairy Food Sci. 2012, 7, 195-201.

7. Dravie, E.E.; Kortei, N.K.; Essuman, E.K.; Tettey, C.O.; Boakye, A.A.; Hunkpe, G. Antioxidant, phytochemical and physicochemical properties of sesame seed (Sesamum indicum L). Sci. Afr. 2020, 8, e00349. [CrossRef]

8. Kim, Y.H.; Kim, E.Y.; Rodriguez, I.; Nam, Y.H.; Jeong, S.Y.; Hong, B.N.; Choung, S.-Y.; Kang, T.H. Sesamum indicum L. Oil and sesamin induce auditory-protective effects through changes in hearing loss-related gene expression. J. Med. Food 2020, 23, 491-498. [CrossRef]

9. Ma'mag, L.K.; Zintchem, A.A.A.; Atchadé, A.d.T.; Kopa Kowa, T.; Tchinda Tiabou, A.; Bikobo, D.S.N.; Pegnyemb, D.E. Antileishmanial activity of long chain alkyl benzene and other constituents from seeds of Sesamum indicum. L (Pedaliaceae). Nat. Prod. Res. 2020, 1-5. [CrossRef]

10. Mekky, R.H.; Abdel-Sattar, E.; Segura-Carretero, A.; Contreras, M.d.M. Phenolic Compounds from Sesame Cake and Antioxidant Activity: A New Insight for Agri-Food Residues' Significance for Sustainable Development. Foods 2019, 8, 432. [CrossRef]

11. Namiki, M. Nutraceutical Functions of Sesame: A Review. Crit. Rev. Food Sci. Nutr. 2007, 47, 651-673. [CrossRef]

12. Hsu, C.-C.; Huang, H.-C.; Wu, P.-T.; Tai, T.-W.; Jou, I.M. Sesame oil improves functional recovery by attenuating nerve oxidative stress in a mouse model of acute peripheral nerve injury: Role of Nrf-2. J. Nutr. Biochem. 2016, 38, 102-106. [CrossRef]

13. Zeb, A.; Muhammad, B.; Ullah, F. Characterization of sesame (Sesamum indicum L.) seed oil from Pakistan for phenolic composition, quality characteristics and potential beneficial properties. J. Food Meas. Charact. 2017, 11, 1362-1369. [CrossRef]

14. Khaleel, A.E.S.; Gonaid, M.H.; El-Bagry, R.I.; Sleem, A.A.; Shabana, M. Chemical and biological study of the residual aerial parts of Sesamum indicum L. J. Food Drug Anal. 2007, 15, 249-257. [CrossRef]

15. Dachtler, M.; van de Put, F.H.M.; Stijn, F.; Beindorff, C.M.; Fritsche, J. On-line LC-NMR-MS characterization of sesame oil extracts and assessment of their antioxidant activity. Eur. J. Lipid Sci. Technol. 2003, 105, 488-496. [CrossRef]

16. Wu, R.; Ma, F.; Zhang, L.; Li, P.; Li, G.; Zhang, Q.; Zhang, W.; Wang, X. Simultaneous determination of phenolic compounds in sesame oil using LC-MS/MS combined with magnetic carboxylated multi-walled carbon nanotubes. Food Chem. 2016, 204, 334-342. [CrossRef] [PubMed]

17. Görgüç, A.; Özer, P.; Yılmaz, F.M. Simultaneous effect of vacuum and ultrasound assisted enzymatic extraction on the recovery of plant protein and bioactive compounds from sesame bran. J. Food Compost. Anal. 2020, 87, 103424. [CrossRef]

18. Görgüç, A.; Bircan, C.; Yllmaz, F.M. Sesame bran as an unexploited by-product: Effect of enzyme and ultrasound-assisted extraction on the recovery of protein and antioxidant compounds. Food Chem. 2019, 283, 637-645. [CrossRef]

19. Shyu, Y.-S.; Hwang, L.S. Antioxidative activity of the crude extract of lignan glycosides from unroasted Burma black sesame meal. Food Res. Int. 2002, 35, 357-365. [CrossRef]

20. Ishtiaque, S.; Khan, N.; Siddiqui, M.A.; Siddiqi, R.; Naz, S. Antioxidant potential of the extracts, fractions and oils derived from oilseeds. Antioxidants 2013, 2, 246-256. [CrossRef]

21. Mekky, R.H.; Contreras, M.d.M.; El-Gindi, M.R.; Abdel-Monem, A.R.; Abdel-Sattar, E.; Segura-Carretero, A. Profiling of phenolic and other compounds from Egyptian cultivars of chickpea (Cicer arietinum L.) and antioxidant activity: A comparative study. RSC Adv. 2015, 5, 17751-17767. [CrossRef] 
22. Mekky, R.H.; Thabet, M.M.; Rodríguez-Pérez, C.; Elnaggar, D.M.Y.; Mahrous, E.A.; Segura-Carretero, A.; Abdel-Sattar, E. Comparative metabolite profiling and antioxidant potentials of seeds and sprouts of three Egyptian cultivars of Vicia faba L. Food Res. Int. 2020, 136, 109537. [CrossRef]

23. Abouzed, T.K.; Contreras, M.d.M.; Sadek, K.M.; Shukry, M.H.; Abdelhady, D.; Gouda, W.M.; Abdo, W.; Nasr, N.E.; Mekky, R.H.; Segura-Carretero, A.; et al. Red onion scales ameliorated streptozotocin-induced diabetes and diabetic nephropathy in Wistar rats in relation to their metabolite fingerprint. Diabetes Res. Clin. Pract. 2018, 140, 253-264. [CrossRef]

24. Ammar, S.; Contreras, M.d.M.; Belguith-Hadrich, O.; Segura-Carretero, A.; Bouaziz, M. Assessment of the distribution of phenolic compounds and contribution to the antioxidant activity in Tunisian fig leaves, fruits, skins and pulps using mass spectrometry-based analysis. Food Funct. 2015, 6, 3663-3677. [CrossRef] [PubMed]

25. Reaxys. Available online: http:/ / www.reaxys.com (accessed on 1 December 2020).

26. KNApSAcK-Core-System. Available online: http://www.knapsackfamily.com/knapsack_jsp/top.html (accessed on 1 December 2020).

27. SciFinder-Scholar. Available online: https://scifinder.cas.org (accessed on 1 December 2020).

28. PubChem. Available online: http://pubchem.ncbi.nlm.nih.gov (accessed on 1 December 2020).

29. ChemSpider. Available online: http:/ / www.chemspider.com (accessed on 1 December 2020).

30. METLIN-Metabolite-Database. Available online: http:/ / metlin.scripps.edu (accessed on 1 December 2020).

31. Phenol-Explorer. Available online: www.phenol-explorer.eu (accessed on 1 December 2020).

32. Dictionary-of-Natural-Products. Available online: http://dnp.chemnetbase.com (accessed on 1 December 2020).

33. Phytochemical-Dictionary-of-Natural-Products. Available online: https://www.crcpress.com/go/the_dictionary_of_natural_ products (accessed on 1 December 2020).

34. Egyptian-Knowledge-Bank. Available online: https://www.ekb.eg/ (accessed on 1 December 2020).

35. Abdel-Sattar, E.; Mahrous, E.A.; Thabet, M.M.; Elnaggar, D.M.Y.; Youssef, A.M.; Elhawary, R.; Zaitone, S.A.; Rodríguez-Pérez, C.; Segura-Carretero, A.; Mekky, R.H. Methanolic extracts of a selected Egyptian Vicia faba cultivar mitigate the oxidative/inflammatory burden and afford neuroprotection in a mouse model of Parkinson's disease. Inflammopharmacology 2020. [CrossRef] [PubMed]

36. Ammar, S.; Contreras, M.d.M.; Belguith-Hadrich, O.; Bouaziz, M.; Segura-Carretero, A. New insights into the qualitative phenolic profile of Ficus carica L. fruits and leaves from Tunisia using ultra-high-performance liquid chromatography coupled to quadrupole-time-of-flight mass spectrometry and their antioxidant activity. RSC Adv. 2015, 5, 20035-20050. [CrossRef]

37. Contreras, M.d.M.; Arráez-Román, D.; Fernández-Gutiérrez, A.; Segura-Carretero, A. Nano-Liquid chromatography coupled to time-of-flight mass spectrometry for phenolic profiling: A case study in cranberry syrups. Talanta 2015, 132, 929-938. [CrossRef]

38. Mekky, R.H. A Comparative Phytochemical and Biological Studies on Certain Egyptian Varieties of Cicer arietinum Linn., Family Fabaceae. Ph.D. Thesis, Cairo University, Cairo, Egypt, 2016.

39. Tsimogiannis, D.; Samiotaki, M.; Panayotou, G.; Oreopoulou, V. Characterization of Flavonoid Subgroups and Hydroxy Substitution by HPLC-MS/MS. Molecules 2007, 12, 593-606. [CrossRef]

40. De Rijke, E.; Out, P.; Niessen, W.M.A.; Ariese, F.; Gooijer, C.; Brinkman, U.A.T. Analytical separation and detection methods for flavonoids. J. Chromatogr. A 2006, 1112, 31-63. [CrossRef]

41. Materska, M. Flavone C-glycosides from Capsicum annuum L.: Relationships between antioxidant activity and lipophilicity. Eur. Food Res. Technol. 2015, 240, 549-557. [CrossRef]

42. Abu-Reidah, I.M.; Arráez-Román, D.; Quirantes-Piné, R.; Fernández-Arroyo, S.; Segura-Carretero, A.; Fernández-Gutiérrez, A. HPLC-ESI-Q-TOF-MS for a comprehensive characterization of bioactive phenolic compounds in cucumber whole fruit extract. Food Res. Int. 2012, 46, 108-117. [CrossRef]

43. Eklund, P.C.; Backman, M.J.; Kronberg, L.Å.; Smeds, A.I.; Sjöholm, R.E. Identification of lignans by liquid chromatographyelectrospray ionization ion-trap mass spectrometry. J. Mass Spectrom. 2008, 43, 97-107. [CrossRef]

44. Guo, H.; Liu, A.-H.; Ye, M.; Yang, M.; Guo, D.-A. Characterization of phenolic compounds in the fruits of Forsythia suspensa by high-performance liquid chromatography coupled with electrospray ionization tandem mass spectrometry. RCM 2007, 21, 715-729.

45. Abu-Reidah, I.M.; Arráez-Román, D.; Segura-Carretero, A.; Fernández-Gutiérrez, A. Extensive characterisation of bioactive phenolic constituents from globe artichoke (Cynara scolymus L.) by HPLC-DAD-ESI-QTOF-MS. Food Chem. 2013, 141, $2269-2277$. [CrossRef] [PubMed]

46. Lee, J.; Choe, E. Extraction of lignan compounds from roasted sesame oil and their effects on the autoxidation of methyl linoleate. J. Food Sci. 2006, 71, C430-C436. [CrossRef]

47. Ammar, S.; Contreras, M.d.M.; Gargouri, B.; Segura-Carretero, A.; Bouaziz, M. RP-HPLC-DAD-ESI-QTOF-MS based metabolic profiling of the potential Olea europaea by-product "wood" and its comparison with leaf counterpart. Phytochem. Anal. 2017, 28, 217-229. [CrossRef]

48. Herzog, K.; IJlst, L.; van Cruchten, A.G.; van Roermund, C.W.T.; Kulik, W.; Wanders, R.J.A.; Waterham, H.R. An UPLC-MS/MS Assay to Measure Glutathione as Marker for Oxidative Stress in Cultured Cells. Metabolites 2019, 9, 45. [CrossRef] [PubMed]

49. Gómez-Romero, M.; Segura-Carretero, A.; Fernández-Gutiérrez, A. Metabolite profiling and quantification of phenolic compounds in methanol extracts of tomato fruit. Phytochemistry 2010, 71, 1848-1864. [CrossRef] 
50. Kozukue, E.; Kozukue, N.; Tsuchida, H. Identification and changes of phenolic compounds in bamboo shoots during storage at $20{ }^{\circ}$ C. J. Jpn. Soc. Hortic. Sci. 1998, 67, 805-811. [CrossRef]

51. Talhaoui, N.; Gómez-Caravaca, A.M.; León, L.; De la Rosa, R.; Fernández-Gutiérrez, A.; Segura-Carretero, A. From Olive Fruits to Olive Oil: Phenolic Compound Transfer in Six Different Olive Cultivars Grown under the Same Agronomical Conditions. Int. J. Mol. Sci. 2016, 17, 337. [CrossRef]

52. De Torres, A.; Espínola, F.; Moya, M.; Alcalá, S.; Vidal, A.M.; Castro, E. Assessment of phenolic compounds in virgin olive oil by response surface methodology with particular focus on flavonoids and lignans. LWT 2018, 90, 22-30. [CrossRef]

53. Miho, H.; Moral, J.; López-González, M.A.; Díez, C.M.; Priego-Capote, F. The phenolic profile of virgin olive oil is influenced by malaxation conditions and determines the oxidative stability. Food Chem. 2020, 314, 126183.

54. Wang, X.; Zeng, Q.; Contreras, M.d.M.; Wang, L. Profiling and quantification of phenolic compounds in Camellia seed oils: Natural tea polyphenols in vegetable oil. Food Res. Int. 2017, 102, 184-194. [CrossRef]

55. Wang, X.; Contreras, M.d.M.; Xu, D.; Xing, C.; Wang, L.; Yang, D. Different distribution of free and bound phenolic compounds affects the oxidative stability of tea seed oil: A novel perspective on lipid antioxidation. LWT 2020, 129, 109389. [CrossRef]

56. Kim, I.; Choe, E. Effects of Bleaching on the Properties of Roasted Sesame Oil. J. Food Sci. 2005, 70, C48-C52. [CrossRef]

57. Wu, S.; Wang, L.; Shu, F.; Cao, W.; Chen, F.; Wang, X. Effect of refining on the lignan content and oxidative stability of oil pressed from roasted sesame seed. Int. J. Food Sci. Technol. 2013, 48, 1187-1192. [CrossRef]

58. Olmo-García, L.; Polari, J.J.; Li, X.; Bajoub, A.; Fernández-Gutiérrez, A.; Wang, S.C.; Carrasco-Pancorbo, A. Deep insight into the minor fraction of virgin olive oil by using LC-MS and GC-MS multi-class methodologies. Food Chem. 2018, 261, 184-193. [CrossRef] [PubMed]

59. Wan, Y.; Li, H.; Fu, G.; Chen, X.; Chen, F.; Xie, M. The relationship of antioxidant components and antioxidant activity of sesame seed oil. J. Sci. Food Agric. 2015, 95, 2571-2578. [CrossRef] [PubMed] 